\title{
Article
}

\section{An Edgeworth Expansion for the Ratio of Two Functionals of Gaussian Fields and Optimal Berry-Esseen Bounds}

\author{
Yoon-Tae Kim and Hyun-Suk Park * \\ Department of Statistics and Data Science Convergence Research Center, Hallym University, \\ Chuncheon 200-702, Korea; ytkim@hallym.ac.kr \\ * Correspondence: hspark@hallym.ac.kr
}

Citation: Kim, Y.-T.; Park, H.-S. An Edgeworth Expansion for the Ratio of Two Functionals of Gaussian Fields and Optimal Berry-Esseen Bounds. Mathematics 2021, 9, 2223. https:/ / doi.org/10.3390/math9182223

Academic Editor: Leonid Piterbarg

Received: 18 August 2021

Accepted: 6 September 2021

Published: 10 September 2021

Publisher's Note: MDPI stays neutral with regard to jurisdictional claims in published maps and institutional affiliations.

Copyright: (C) 2021 by the authors. Licensee MDPI, Basel, Switzerland. This article is an open access article distributed under the terms and conditions of the Creative Commons Attribution (CC BY) license (https:// creativecommons.org/licenses/by/ $4.0 /)$.
Abstract: This paper is concerned with the rate of convergence of the distribution of the sequence $\left\{F_{n} / G_{n}\right\}$, where $F_{n}$ and $G_{n}$ are each functionals of infinite-dimensional Gaussian fields. This form very frequently appears in the estimation problem of parameters occurring in Stochastic Differential Equations (SDEs) and Stochastic Partial Differential Equations (SPDEs). We develop a new technique to compute the exact rate of convergence on the Kolmogorov distance for the normal approximation of $F_{n} / G_{n}$. As a tool for our work, an Edgeworth expansion for the distribution of $F_{n} / G_{n}$, with an explicitly expressed remainder, will be developed, and this remainder term will be controlled to obtain an optimal bound. As an application, we provide an optimal Berry-Esseen bound of the Maximum Likelihood Estimator (MLE) of an unknown parameter appearing in SDEs and SPDEs.

Keywords: Malliavin calculus; fourth moment theorem; Kolmogorov distance; multiple stochastic integral; Stein's equation; Edgeworth expansion; stochastic (partial) differential equations; BerryEsseen bound

\section{Introduction}

Let $X=\{X(h), h \in \mathfrak{H}\}$ be an isonormal Gaussian process defined on a probability space $(\Omega, \mathfrak{F}, \mathbb{P})$, where $\mathfrak{H}$ is a real separable Hilbert space, and let $\left\{F_{n}, n \geq 1\right\}$ be a sequence of random variables of functionals of infinite-dimensional Gaussian fields associated with $X$. In recent years, there have been many efforts to find necessary and sufficient conditions for a normal approximation. The authors in [1] discovered a surprising central limit theorem, called the "fourth moment theorem", for a sequence of random variables belonging to some Wiener chaos associated with $X$.

Theorem 1 (Fourth moment theorem). Fix an integer $q \geq 2$ and consider a sequence of random variables $\left\{F_{n}, n \geq 1\right\}$ belonging to the qth Wiener chaos with $\mathbb{E}\left[F_{n}^{2}\right]=1$ for all $n \geq 1$. Then $F_{n} \stackrel{\mathcal{L}}{\longrightarrow} Z$ if and only if $\mathbb{E}\left[F_{n}^{4}\right] \rightarrow 3=\mathbb{E}\left[Z^{4}\right]$, where $Z$ is a standard Gaussian random variable and the notation $\stackrel{\mathcal{L}}{\longrightarrow}$ denotes the convergence in distribution.

Afterwards, the authors in [2] obtained the upper bound of the distances between two random variable $F_{n}$ and $Z$ by using the techniques based on the combination between Malliavin calculus (see, e.g., [3-6]) and Stein's method for normal approximation (see, e.g., [7-9]). For instance, in the case of the Kolmogorov distance $d_{K o l}$, one obtained that

$$
d_{K o l}\left(F_{n}, Z\right) \leq \sqrt{\mathbb{E}\left[\left(1-\left\langle D F_{n},-D L^{-1} F_{n}\right\rangle_{\mathfrak{H}}\right)^{2}\right]}:=\varphi\left(F_{n}\right)
$$

where $D F_{n}$ and $L^{-1}$ denote the Malliavin derivative of $F_{n}$ and the pseudo-inverse of the Ornstein-Uhlhenbeck generator, respectively (see Section 2). In the particular case where $F_{n}$ is 
an element in the $q$ th Wiener chaos of $X$ with $\mathbb{E}\left[F_{n}^{2}\right]=1$, the upper bound (1) is expressed in the following form

$$
d_{K o l}\left(F_{n}, Z\right) \leq \sqrt{\frac{q-1}{3 q}\left(\mathbb{E}\left[F_{n}^{4}\right]-3\right)},
$$

where $\mathbb{E}\left[F_{n}^{4}\right]-3$ is just the fourth cumulant of $F_{n}$. Recall that the bound $\varphi\left(F_{n}\right)$ is optimal for the sequence $\left\{F_{n}\right\}$ with respect to some distance $d$ if there exist constants $0<c<C<\infty$, independent of $n$, such that

$$
c \varphi\left(F_{n}\right) \leq d\left(F_{n}, Z\right) \leq C \varphi\left(F_{n}\right), \text { for all } n \geq 1 .
$$

In [10], the authors prove that whenever the distance $d$ is defined as the supremum over a class of twice continuously differentiable functions with a bounded second derivative, an optimal rate of convergence is given by the sequence

$$
\max \left\{\left|\mathbb{E}\left[F_{n}^{3}\right]\right|,\left|\mathbb{E}\left[F_{n}^{4}\right]-3\right|\right\}, n \geq 1
$$

in the case where $\left\{F_{n}\right\}$ is a sequence of random variables belonging to a fixed Wiener chaos. The further work in [11] subsequent to this result is that the optimal rate in the total variation distance exactly coincides with the rate in the case of the distance related to the smooth test functions. When we considered the statistical estimation problem of parameters appearing in SDEs or SPDEs, quite often we encountered statistics of the form $F_{n} / G_{n}$. In these circumstances, how an upper bound $\varphi(n)$ in (1) is derived can not be applied directly. The authors of [12] developed a technique to obtain the upper and lower rates of the convergence on the Kolmogorov distance for a sequence $\left\{F_{n} / G_{n}\right\}$. Using both these rates, the authors obtain the optimal bound for the convergence rate of the normal approximation for MLE of the parameter occurring in SPDE. However, the paper [12] does not provide a complete answer to the optimal rates of convergence on the Kolmogorov distance because the convergence rates in the upper and lower bound derived in [12] may not match each other.

The aim of the present work is to find a complete answer for the optimal rate. To this end, a set of sufficient conditions will be derived, ensuring that the upper and lower bounds yield an optimal rate for the Kolmogorov distance. Our methodology to obtain the optimal bound is based on an Edgeworth expansion of $\mathbb{P}\left(F_{n} / G_{n} \leq z\right)$ derived from that for $\mathbb{P}\left(F_{n} \leq z\right)$ in [13] (for Edgeworth expansions, see, e.g., Chapter 2 in [14] and Chapter 5 in [15]). In that paper, the Edgeworth expansion with general order $J$ is given by

$$
\mathbb{P}\left(F_{n} \leq z\right)-\mathbb{P}(Z \leq z)=\sum_{s=2}^{J} H_{s}(z) \phi(z) \mathfrak{g}_{s}\left(F_{n}\right)+R_{z}^{(J+1)}\left(F_{n}\right),
$$

where the quantity $\mathfrak{g}_{s}\left(F_{n}\right)$ can be expressed in terms of the cumulants of $F_{n}, H_{s}(z)$ is the Hermite polynomial of order $s$, and $\phi$ is the probability density function of a standard Gaussian distribution. It is easier and more extensive for newly developed techniques to apply to the estimation problems for parameters occurring in SDEs or SPDEs than to existing ones. Moreover, a set of sufficient conditions derived in this work is satisfied in most of the estimation problems for parameters in SDEs and SPDEs.

The rest of the paper is organized as follows. Section 2 reviews some basic notations, and the results of Malliavin calculus and Stein's method. In Section 3, the Edgeworth expansion with general order will be derived. Using this expansion, an optimal bound in Kolmogorov distance will be obtained in Section 4. Finally, as an application of our main results, in Section 5, we provide an optimal Berry-Esseen bound for the estimator of parameters in SDE and SPDE.

Throughout this paper, $c$ (or $C$ ) stands for an absolute constant with possibly different values in different places. 


\section{Preliminaries}

\subsection{Malliavin Calculus}

In this section, we recall some basic facts about Malliavin calculus for Gaussian processes. The reader is referred to $[5,6]$ for a more detailed explanation. Suppose that $\mathfrak{H}$ is a real separable Hilbert space with scalar product denoted by $\langle\cdot, \cdot\rangle_{\mathfrak{H}}$. Let $B=\{B(h), h \in \mathfrak{H}\}$ be an isonormal Gaussian process, that is a centered Gaussian family of random variables such that $\mathbb{E}[B(h) B(g)]=\langle h, g\rangle_{\mathfrak{H}}$. For every $n \geq 1$, let $\mathbb{H}_{n}$ be the $n$th Wiener chaos of $B$, that is the closed linear subspace of $\mathbb{L}^{2}(\Omega)$ generated by $\left\{H_{n}(B(h)): h \in \mathfrak{H},\|h\|_{\mathfrak{H}}=1\right\}$, where $H_{n}$ is the $n$th Hermite polynomial. We define a linear isometric mapping $I_{n}: \mathfrak{H}^{\odot n} \rightarrow \mathbb{H}_{n}$ by $I_{n}\left(h^{\otimes n}\right)=n ! H_{n}(B(h))$, where $\mathfrak{H}^{\odot n}$ is the symmetric tensor product. It is well known that any square integrable random variable $F \in L^{2}(\Omega, \mathcal{G}, \mathbb{P})(\mathcal{G}$ denotes the $\sigma$-field generated by $B)$ can be expanded into a series of multiple stochastic integrals:

$$
F=\sum_{q=0}^{\infty} I_{q}\left(f_{q}\right)
$$

where $f_{0}=\mathbb{E}[F]$, the series converges in $L^{2}$, and the functions $f_{q} \in \mathfrak{H}^{\odot q}$ are uniquely determined by $F$. Let $\left\{e_{l}, l \geq 1\right\}$ be a complete orthonormal system in $\mathfrak{H}$. If $f \in \mathfrak{H}^{\odot p}$ and $g \in \mathfrak{H}^{\odot q}$, the contraction $f \otimes_{r} g, 1 \leq r \leq p \wedge q$, is the element of $\mathfrak{H}^{\otimes(p+q-2 r)}$, defined by

$$
f \otimes_{r} g=\sum_{l_{1}, \cdots, l_{r}=1}^{\infty}\left\langle f, e_{l_{1}} \otimes \cdots \otimes e_{l_{r}}\right\rangle_{\mathfrak{H} \otimes r} \otimes\left\langle g, e_{l_{1}} \otimes \cdots \otimes e_{l_{r}}\right\rangle_{\mathfrak{H}^{\otimes r}}
$$

The following formula for the product of the multiple stochastic integrals will be frequently used.

Proposition 1. Let $f \in \mathfrak{H}^{\odot p}$ and $g \in \mathfrak{H}^{\odot q}$ be two symmetric functions. Then

$$
I_{p}(f) I_{q}(g)=\sum_{r=0}^{p \wedge q} r !\left(\begin{array}{l}
p \\
r
\end{array}\right)\left(\begin{array}{l}
q \\
r
\end{array}\right) I_{p+q-2 r}\left(f \otimes_{r} g\right) .
$$

Let $\mathfrak{S}$ be the class of smooth and cylindrical random variables $F$ of the form

$$
F=f\left(B\left(\varphi_{1}\right), \cdots, B\left(\varphi_{n}\right)\right),
$$

where $n \geq 1, f \in \mathcal{C}_{b}^{\infty}\left(\mathbb{R}^{n}\right)$ and $\varphi_{i} \in \mathfrak{H}, i=1, \cdots, n$. The Malliavin derivative of $F$ with respect to $B$ is the element of $L^{2}(\Omega, \mathfrak{H})$, defined by

$$
D F=\sum_{i=1}^{n} \frac{\partial f}{\partial x_{i}}\left(B\left(\varphi_{1}\right), \cdots, B\left(\varphi_{n}\right)\right) \varphi_{i} .
$$

We denote by $\mathbb{D}^{l, p}$ the closure of its associated smooth random variable class with respect to the norm

$$
\|F\|_{l, p}^{p}=\mathbb{E}\left(|F|^{p}\right)+\sum_{k=1}^{l} \mathbb{E}\left(\left\|D^{k} F\right\|_{\mathfrak{H}^{\otimes k}}^{p}\right) .
$$

We denote by $\delta$ the adjoint of the operator $D$, also called the divergence operator. The domain of $\delta$, denoted by $\operatorname{Dom}(\delta)$, is an element $u \in \mathbb{L}^{2}(\Omega ; \mathfrak{H})$ such that

$$
\left|\mathbb{E}\left(<D^{l} F, u>_{\mathfrak{H}^{\otimes l}}\right)\right| \leq C\left(\mathbb{E}|F|^{2}\right)^{1 / 2} \text { for all } F \in \mathbb{D}^{l, 2} .
$$

If $u \in \operatorname{Dom}(\delta)$, then $\delta(u)$ is the element of $L^{2}(\Omega)$ defined by the duality relationship

$$
\mathbb{E}[F \delta(u)]=\mathbb{E}\left[\langle D F, u\rangle_{\mathfrak{H}}\right] \text { for every } F \in \mathbb{D}^{1,2} .
$$


Let $F \in L^{2}(\Omega)$ be a square integrable random variable. The operator $L$ is defined through the projection operator $J_{n}, n=0,1,2 \ldots$, as $L=\sum_{n=0}^{\infty}-n J_{n} F$, and is called the infinitesimal generator of the Ornstein-Uhlhenbeck semigroup. The relationship between the operator $D, \delta$, and $L$ is given as follows: $\delta D F=-L F$; that is, for $F \in L^{2}(\Omega)$ the statement $F \in$ $\operatorname{Dom}(L)$ is equivalent to $F \in \operatorname{Dom}(\delta D)$ (i.e. $F \in \mathbb{D}^{1,2}$ and $D F \in \operatorname{Dom}(\delta)$ ), and in this case $\delta D F=-L F$. We also define the operator $L^{-1}$, which is the pseudo-inverse of $L$, as $L^{-1} F=$ $\sum_{n=1}^{\infty} \frac{1}{n} J_{n}(F)$. Note that $L^{-1}$ is an operator with values in $\mathbb{D}^{2,2}$ and $L L^{-1} F=F-\mathbb{E}[F]$ for all $F \in L^{2}(\Omega)$.

\subsection{Stein's Method}

In this paper, we focus only on the normal approximation of random variables with respect to the Kolmogorov distance defined as

$$
d_{\text {Kol }}(F, Z)=\sup _{z \in \mathbb{R}}|\mathbb{P}(X \leq z)-\mathbb{P}(Z \leq z)|
$$

where $Z$ is a standard Gaussian random variable. For fixed $z \in \mathbb{R}$, we consider the Stein equation

$$
f^{\prime}(x)-x f(x)=\mathbf{1}_{(-\infty, z]}(x)-\Phi(z),
$$

where $\Phi(z)=\mathbb{P}(Z \leq z)$. It is well known (see, e.g., [7]) that for every fixed $z \in \mathbb{R}$, the function

$$
\begin{aligned}
f_{z}(x) & =e^{x^{2} / 2} \int_{-\infty}^{x}\left[\mathbf{1}_{(-\infty, z]}(u)-\Phi(z)\right] e^{-u^{2} / 2} d u \\
& = \begin{cases}\sqrt{2 \pi} e^{x^{2} / 2} \Phi(x)(1-\Phi(z)) & \text { if } x \leq z \\
\sqrt{2 \pi} e^{x^{2} / 2} \Phi(z)(1-\Phi(x)) & \text { if } x>z,\end{cases}
\end{aligned}
$$

is a solution to the Stein Equation (9) such that $\left\|f_{z}\right\|_{\infty} \leq \sqrt{2 \pi} / 4$ and $\left\|f_{z}^{\prime}\right\|_{\infty} \leq 1$. For given function $h: \mathbb{R} \rightarrow \mathbb{R}$ such that $\mathbb{E}[|h(Z)|]<\infty$, we define

$$
\left(U_{Z} h\right)(w)=e^{\frac{1}{2} w^{2}} \int_{-\infty}^{w}(h(x)-\mathbb{E}[h(Z)]) e^{-\frac{1}{2} x^{2}} d x,
$$

and write $\left(U_{N} \mathbf{1}_{(-\infty, z]}\right)(w)=f_{z}(w)$.

The next lemma gives some properties of the function $U_{Z} h$ (see, e.g., [5]).

Lemma 1 (Stein's equation). Given a bounded function $h: \mathbb{R} \rightarrow \mathbb{R}$, there exists an absolutely continuous function $U_{Z}$ h solving the Stein's equation:

$$
f^{\prime}(x)-x f(x)=h(x)-\mathbb{E}[h(Z)] \text { for all } x \in \mathbb{R}
$$

for all $x$ and satisfying

$$
\left\|U_{Z} h\right\|_{\infty} \leq \sqrt{\frac{\pi}{2}}\|h-\mathbb{E}[h(Z)]\|_{\infty} \text { and }\left\|\left(U_{Z} h\right)^{\prime}\right\|_{\infty} \leq 2\|h-\mathbb{E}[h(Z)]\|_{\infty} .
$$

We define a function

$$
\left(U_{Z g}\right)^{(i)}(x)= \begin{cases}\left(U_{Z} g\right)(x) & \text { for } i=0 \\ \left(U_{Z g}\right)^{\prime}(x) & \text { for } i=1 .\end{cases}
$$

Let us set

$$
\left(\Psi_{i_{1}, \ldots, i_{k} g} g\right)(x)=\underbrace{\left(U_{Z}\left(\cdots\left(U_{Z}\left(U_{Z} g\right)^{\left(i_{1}\right)}\right)^{\left(i_{2}\right)} \cdots\right)^{\left(i_{k}\right)}\right.}_{k}(x) \text { for } i_{1}, \ldots, i_{k} \in\{0,1\} .
$$


We shall denote by $H_{q}$ the $q$ th Hermite polynomial defined as follows: $H_{0}(x)=1$ and for $q \geq 1$

$$
H_{q}(x)=(-1)^{q} e^{\frac{1}{2} x^{2}} \frac{d^{q}}{d x^{q}} e^{-\frac{1}{2} x^{2}}, x \in \mathbb{R} .
$$

\section{Edgeworth Expansion}

Now we derive the $J$ th order Edgeworth expansion of $\mathbb{P}(F / G \leq z)$ for $z \in \mathbb{R}$. We begin by the simple result, given in Lemma 2.3 in [10].

Lemma 2. Suppose that $F \in \mathbb{D}^{1,2}$ and $\mathbb{E}\left[|G|^{2}\right]<\infty$. Then $L^{-1} G \in \mathbb{D}^{2,2}$ and

$$
\mathbb{E}\left[\left\langle D F,-D L^{-1} G\right\rangle_{\mathfrak{H}}\right]=\mathbb{E}[F G]-\mathbb{E}[F] \mathbb{E}[G] .
$$

Before stating the Edgeworth expansion, we introduce some notations including the Gamma operators $\Gamma$ (see [16] for a more detailed explanation). Let $F \in \mathbb{D}^{1,2}$. Define $\Gamma_{0}(F)=F$ and $\Gamma_{1}(F, F)=\left\langle D F,-D L^{-1} F\right\rangle_{\mathfrak{H}}$, and we write

$$
\Gamma_{j}(F)=\left\langle D F,-D L^{-1} \Gamma_{j-1}(F)\right\rangle_{\mathfrak{H}}, \text { for } j=0,1, \ldots
$$

Similarly, let $F \in \mathbb{D}^{1,2}$, we define $\Gamma_{0}^{*}(F)=F$ and $\Gamma_{1}^{*}(F)=\Gamma_{1}(F)$. If $\Gamma_{j}^{*}(F) \in \mathbb{D}^{1,2}$ for fixed $j \leq 1$, we write

$$
\Gamma_{j+1}^{*}(F)=\left\langle-D L^{-1} F, D \Gamma_{j}^{*}(F)\right\rangle_{\mathfrak{H}} .
$$

Let $F \in \mathbb{D}^{j, 2^{j}}$ for a fixed integer $j \geq 1$. Then, for all $k=1, \ldots, j$, it is easy, from Lemma 4.2 in [16], to see that $\Gamma_{k}^{*}(F)$ is a well-defined element in $\mathbb{D}^{j-k, 2^{j-k}}$, where we set by convention $\mathbb{D}^{0,1}=L^{1}(\Omega)$. For $F \in \mathbb{D}^{1,2}$, define an operator $D_{F}: \mathbb{D}^{1,2} \rightarrow L^{2}(\Omega)$ by $D_{F}^{0}(G)=G$,

$$
D_{F}(G)=\left\langle-D L^{-1} F, D G\right\rangle_{\mathfrak{H}} \in L^{1}(\Omega)
$$

and for $F, G \in \mathbb{D}^{k, 2^{k}}$, we define

$$
D_{F}^{k}(G)=\underbrace{D_{F}\left(D _ { F } \left(\cdots \left(D_{F}\right.\right.\right.}_{k}(G)) \cdots) \in L^{1}(\Omega) .
$$

Let us define two sets $\mathfrak{D}(r, k)$ and $\mathfrak{D}_{\alpha, \beta}^{*}(r, k)$ for $r=1,2, \ldots$ and $k=1, \ldots$ by

$$
\mathfrak{D}(r, k)=\left\{\left(i_{1}, \ldots, i_{k}\right) \in\{0,1\}^{k}: \sum_{l=1}^{k} i_{l}+k+1=r\right\},
$$

and, for $\alpha=-1,1,2$ and $\beta=1,2$,

$$
\mathfrak{D}_{(\alpha, \beta)}^{*}(J, k+1)=\left\{\left(i_{1}, \ldots, i_{k+1}\right) \in \mathfrak{D}(J+\beta, k+1):\left(i_{1}, \ldots, i_{k}\right) \in \mathfrak{D}(J+\alpha, k)\right\},
$$

respectively. For the sake of simplicity, we write

$$
\bar{\Gamma}_{1}(F)^{m}:=\left\{1-\Gamma_{1}(F)\right\}^{m},
$$


and sometimes abbreviate the argument $F$ in the operator $\bar{\Gamma}_{1}(F)^{m}$ for $m=0,1$ and $\Gamma_{j}^{*}(F)$ for $j=2, \ldots$. Let us set

$$
\begin{aligned}
\mathfrak{g}_{1}(F)= & \mathbb{E}\left[\bar{\Gamma}_{1}(F)\right], \\
\mathfrak{g}_{s}(F)= & \frac{1}{s+1} \sum_{k=\left\lceil\frac{s-1}{2}\right\rceil}^{s-1} \sum_{i_{1}, \ldots, i_{k} \in \mathfrak{D}(s, k)}(-1)^{\sum_{l=1}^{k} i_{l}+2 k} \\
& \quad \times \prod_{l=1}^{k} \frac{1}{\sum_{r=l}^{k} i_{r}+(k-l+1)} \\
& \quad \times \mathbb{E}\left[D_{F}^{1-i_{k}}\left(\bar{\Gamma}_{1}^{i_{k}} D_{F}^{1-i_{k-1}}\left(\bar{\Gamma}_{1}^{i_{k-1}} \cdots\left(\bar{\Gamma}_{1}^{i_{3}} D_{F}^{1-i_{2}}\left(\bar{\Gamma}_{1}^{i_{2}} D_{F}^{1-i_{1}}\left(\bar{\Gamma}_{1}^{i_{1}+1}\right)\right)\right) \cdots\right)\right]\right.
\end{aligned}
$$

for $s \geq 2$, where the notation $\lceil\cdot\rceil$ denotes the ceiling function defined by

$$
\lceil x\rceil=\inf \{n \in \mathbb{N}: x \leq n\} .
$$

Let us define

$$
\begin{aligned}
Q_{i_{1}, \ldots, i_{k}}\left(A_{z}\right)= & (-1)^{\sum_{l=1}^{k} i_{l}+k} \mathbb{E}\left[\Psi_{i_{1}, \ldots, i_{k}} f_{z}^{\prime}\left(A_{z}\right)\right. \\
& \left.\times D_{F}^{1-i_{k}}\left(\bar{\Gamma}_{1}^{i_{k}} D_{A_{z}}^{1-i_{k-1}} \cdots\left(\bar{\Gamma}_{1}^{i_{3}} D_{A_{z}}^{1-i_{2}}\left(\bar{\Gamma}_{1}^{i_{2}} D_{A_{z}}^{1-i_{1}}\left(\bar{\Gamma}_{1}^{i_{1}+1}\right)\right)\right) \cdots\right)\right] .
\end{aligned}
$$

By just following the proof of Theorem 2 in [13], we obtain an Edgeworth expansion of $F / G$.

Theorem 2 (Edgeworth Expansion). Let $F, G \in \mathbb{D}^{I+2,2^{2+2}}$ have $\mathbb{E}[F]=0$ and $G>0$ a.s. Moreover, assume that $F$ and $G$ have an absolutely continuous law with respect to the Lebesgue measure. Then we have that, for $z \in \mathbb{R}$,

$$
\begin{aligned}
& \mathbb{P}\left(\frac{F}{G} \leq z\right)-\mathbb{P}(Z \leq z) \\
= & \sum_{s=1}^{J} H_{s}(z) \phi(z) \mathfrak{g}_{s}\left(A_{z}\right)+R_{z}^{(J+1)}\left(A_{z}\right),
\end{aligned}
$$

where

$$
A_{z}=\frac{F-z \bar{G}}{\mathbb{E}[G]}, \bar{G}=G-\mathbb{E}[G],
$$

and the remainder term is given by

$$
\begin{aligned}
R_{z}^{(J+1)}\left(A_{z}\right)= & \sum_{\left(i_{1}, \ldots, i_{\left\lceil\frac{I-1}{2}\right\rceil}\right) \in \mathfrak{D}\left(J+1,\left\lceil\frac{J-1}{2}\right\rceil\right)} Q_{\left.i_{1}, \ldots, i_{\lceil} \frac{I-1}{2}\right\rceil}\left(A_{z}\right) \\
& +\sum_{k=\left\lceil\frac{I-1}{2}\right\rceil}^{J-1} \sum_{\left(i_{1}, \ldots, i_{k+1}\right) \in \cup_{\beta=1}^{2} \mathfrak{D}_{(0, \beta)}^{*}(J, k+1)} Q_{i_{1}, \ldots, i_{k+1}}\left(A_{z}\right) \\
& +\sum_{k=\left\lceil\frac{I-2}{2}\right\rceil}^{J-2} \sum_{\left(i_{1}, \ldots, i_{k+1}\right) \in \mathfrak{D}_{(-1,1)}^{*}(J, k+1)} Q_{i_{1}, \ldots, i_{k+1}}\left(A_{z}\right) .
\end{aligned}
$$

Here, if $J$ is odd, then $\mathfrak{D}\left(J+1,\left\lceil\frac{J-1}{2}\right\rceil\right)=\varnothing$. In this case, the first term in $R_{z}^{(J+1)}\left(A_{z}\right)$ has vanished.

Proof. Since $G>0$ a.s., we have that

$$
\mathbb{P}\left(\frac{F}{G} \leq z\right)=\mathbb{P}\left(\frac{F-z(G-\mathbb{E}[G])}{\mathbb{E}[G]} \leq z\right) .
$$


For fixed $z \in \mathbb{R}$, obviously, $A_{z} \in \mathbb{D}^{1,2}$ with $\mathbb{E}\left[A_{z}\right]=0$, and has an absolutely continuous law with respect to Lebesgue measure. By Stein's equation, we deduce, from (17), that, for every $z \in \mathbb{R}$,

$$
\begin{aligned}
\mathbb{P}\left(\frac{F}{G} \leq z\right)-\mathbb{P}(Z \leq z) & =\mathbb{P}\left(A_{z} \leq z\right)-\mathbb{P}(Z \leq z) \\
& =\mathbb{E}\left[f_{z}^{\prime}\left(A_{z}\right)-A_{z} f_{z}\left(A_{z}\right)\right]
\end{aligned}
$$

Applying the iterared method in the proof of Theorem 2 of the paper [13] to the expectation of (18), we get

$$
\mathbb{P}\left(\frac{F}{G} \leq z\right)-\mathbb{P}(Z \leq z)=\sum_{s=1}^{J} H_{s}(z) \phi(z) \mathfrak{g}_{s}\left(A_{z}\right)+R_{z}^{(J+1)}\left(A_{z}\right) .
$$

Hence the proof of this theorem is completed.

Before stating and proving the main results in this section, we need to recall the multi-index notation. A multi-index is a vector of non-negative integers of the type $m=\left(m_{1}, \ldots m_{d}\right) \in \mathbb{N}^{d}$. Then we write

$$
|m|=\sum_{i=1}^{d} m_{i}, \quad \partial_{i}=\frac{\partial}{\partial_{x_{i}}}, \quad \partial^{m}=\partial_{1}^{m_{1}} \ldots \partial_{d}^{m_{d}}, \quad x^{m}=\prod_{i=1}^{d} x_{i}^{m_{i}} .
$$

For convention, we set $0^{0}=1$. For $n \geq 1$, we consider a vector of real-valued random variables $X_{[d]}=\left(X_{1}, \ldots, X_{d}\right)$ such that $\mathbb{E}\left[\left|X_{i}\right|^{d}\right]<\infty$ for every $i=1, \ldots d$. For every $b=\left\{i_{1}, \ldots, i_{k}\right\} \subset[d]=\{1, \ldots, d\}$, we write

$$
X_{b}=\left(X_{i_{1}}, \ldots, X_{i_{k}}\right) \text { and } X^{b}=X_{i_{1}} \cdots X_{i_{k}} .
$$

The cumulant of the vector $X_{b}$ is defined as

$$
\kappa\left(X_{b}\right)=\left.(-i)^{k} \frac{\partial^{k}}{\partial t_{1} \ldots \partial t_{k}} \log \mathbb{E}\left[\exp \left(i \sum_{\ell=1}^{k} t_{\ell} X_{i_{\ell}}\right)\right]\right|_{t_{1}=\cdots=t_{k}=0} .
$$

Definition 1. Let $X_{[d]}=\left(X_{1}, \ldots, X_{d}\right)$ be a $\mathbb{R}^{d}$-valued random vector with $E\left[|X|^{m}\right]<\infty$ for some multi-index $m \in \mathbb{N}^{d} \backslash\{0\}$. The joint cumulant of order $m$ of $X$ is defined as

$$
\kappa_{m}\left(X_{1}, \ldots, X_{d}\right)=\left.(-i)^{|m|} \partial^{m} \log \mathbb{E}\left[\exp \left(i \sum_{\ell=1}^{d} t_{\ell} X_{\ell}\right)\right]\right|_{t_{1}=\cdots=t_{d}=0}
$$

For $m=\left(m_{1}, \ldots m_{d}\right)$, we have that

$$
\kappa_{m}(X)=\kappa(\underbrace{x_{1}, \ldots, X_{1}}_{m_{1}}, \underbrace{X_{2}, \ldots, X_{2}}_{m_{2}}, \ldots, \underbrace{X_{d}, \ldots X_{d}}_{m_{d}}) .
$$

Next, we obtain $J$-th order, $J=1,2$, an Edgeworth expansion by expressing $\mathfrak{g}_{s}, s=1,2$, in terms of the cumulants of $(F, \bar{G})$. For $X_{[m]}=\left(X_{1}, \ldots, X_{m}\right)$, we write $\kappa\left(X_{[m]}\right)=\kappa_{m}(X)$ if $X_{i}=X$ for $i=1, \ldots, m$. The next result contains the relation between cumulants and the moment associated with $X_{[m]}$ (see, e.g., [17]). 
Lemma 3. For every $b \subseteq[n]$,

$$
\begin{aligned}
\mathbb{E}\left[X^{b}\right] & =\sum_{\pi=\left\{b_{1}, \ldots, b_{k}\right\} \in \mathcal{P}(b)} \prod_{i=1}^{k} \kappa\left(X_{b_{i}}\right), \\
\kappa\left(X_{b}\right) & =\sum_{\pi=\left\{b_{1}, \ldots, b_{k}\right\} \in \mathcal{P}(b)}(-1)^{k-1}(k-1) \prod_{i=1}^{k} \mathbb{E}\left[X^{a_{i}}\right] .
\end{aligned}
$$

The following result can be obtained from Lemma 6 in [13].

Lemma 4. Let $F$ and $G$ be random variables with $F, G \in \mathbb{D}^{3,2^{3}}$ with $\mathbb{E}[F]=0$. Then

$$
\mathfrak{g}_{1}\left(A_{z}\right)=-\frac{1}{2 !} \kappa_{2}\left(A_{z}\right), \mathfrak{g}_{2}\left(A_{z}\right)=-\frac{1}{3 !} \kappa_{3}\left(A_{z}\right), \mathfrak{g}_{3}\left(A_{z}\right)=-\frac{1}{4 !} \kappa_{4}\left(A_{z}\right) .
$$

It is immediate, from Theorem 2 and Lemma 4 , that the expansions with an explicit remainder will be given in the case where $J=1,2$. Let us set $X=(F, \bar{G}), e_{1}=(1,0)$ and $e_{2}=(0,1)$.

Corollary 1. Under the assumptions in Theorem 2, we have

(a) for $J=1$,

$$
\begin{aligned}
& \mathbb{P}\left(\frac{F}{G} \leq z\right)-\mathbb{P}(Z \leq z) \\
= & \frac{H_{1}(z) \phi(z)}{2(\mathbb{E}[G])^{2}}\left\{(\mathbb{E}[G])^{2}-\sum_{\alpha=0}^{2}\left(\begin{array}{c}
2 \\
\alpha
\end{array}\right)(-z)^{\alpha} \mathcal{K}_{(2-\alpha) e_{1}+\alpha e_{2}}(X)\right\} \\
& +R_{z}^{(2)}\left(A_{z}\right)
\end{aligned}
$$

where the remainder term $R_{z}^{(2)}\left(A_{z}\right)$ is given by

$$
R_{z}^{(2)}\left(A_{z}\right)=\mathbb{E}\left[\Psi_{1} f_{z}^{\prime}\left(A_{z}\right) \bar{\Gamma}_{1}^{2}\left(A_{z}\right)\right]+\mathbb{E}\left[\Psi_{0} f_{z}^{\prime}(F) \Gamma_{2}^{*}\left(A_{z}\right)\right] .
$$

(b) for $J=2$,

$$
\begin{aligned}
& \mathbb{P}\left(\frac{F}{G} \leq z\right)-\mathbb{P}(Z \leq z) \\
= & \frac{H_{1}(z) \phi(z)}{2(\mathbb{E}[G])^{2}}\left\{(\mathbb{E}[G])^{2}-\sum_{\alpha=0}^{2}\left(\begin{array}{c}
2 \\
\alpha
\end{array}\right)(-z)^{\alpha} \mathcal{K}_{(2-\alpha) e_{1}+\alpha e_{2}}(X)\right\} \\
& -\frac{H_{2}(z) \phi(z)}{3 !(\mathbb{E}[G])^{3}} \sum_{\alpha=0}^{3}\left(\begin{array}{c}
3 \\
\alpha
\end{array}\right)(-z)^{\alpha} \kappa_{(3-\alpha) e_{1}+\alpha e_{2}}(X) . \\
& +R_{z}^{(3)}\left(A_{z}\right)
\end{aligned}
$$

where the remainder term $R_{z}^{(3)}\left(A_{z}\right)$ is given by

$$
\begin{aligned}
R_{z}^{(3)}\left(A_{z}\right)= & \mathbb{E}\left[\Psi_{1} f_{z}^{\prime}\left(A_{z}\right) \bar{\Gamma}_{1}^{2}\left(A_{z}\right)\right]+2 \mathbb{E}\left[\Psi_{0,1} f_{z}^{\prime}(F) \bar{\Gamma}_{1}\left(A_{z}\right) \Gamma_{2}^{*}\left(A_{z}\right)\right] \\
& -\mathbb{E}\left[\Psi_{0,0} f_{z}^{\prime}(F) \Gamma_{3}^{*}\left(A_{z}\right)\right]
\end{aligned}
$$

Proof. By homogeneity and multilinearity of the joint cumulant, we get

$$
\kappa_{q}\left(A_{z}\right)=\frac{1}{(\mathbb{E}[G])^{q}} \sum_{r=0}^{q}\left(\begin{array}{l}
q \\
r
\end{array}\right)(-z)^{q-r} \kappa(\underbrace{F, \ldots, F}_{r}, \underbrace{\bar{G}, \ldots, \bar{G}}_{q-r}) .
$$


Using the formula (27) yields

$$
\begin{aligned}
& \left.\kappa_{2}\left(A_{z}\right)=\frac{1}{(\mathbb{E}[G])^{2}}\left(\kappa_{2}(F)+2(-z) \kappa(F, \bar{G})+z^{2} \kappa_{2}(\bar{G})\right)\right) \\
& \kappa_{3}\left(A_{z}\right)=\frac{1}{(\mathbb{E}[G])^{3}}\left(\kappa_{3}(F)-3 z \kappa(F, F, \bar{G})+3 z^{2} \kappa(F, \bar{G}, \bar{G})+z^{3} \kappa_{3}(\bar{G})\right) .
\end{aligned}
$$

By using Theorem 2 and Lemma 4, together with (28) and (29), we get the expansions (23) and (25).

Next, by using a formula for computing joint cumulants of the multiple stochastic integrals, the Edgeworth expansion of $F / G$, in the case where $F=I_{2}(f)$ and $\bar{G}=I_{2}(g)$, will be derived. For this, we introduce the diagrams and related notations (see [18] for a more detailed explanation). We will denote by $\Gamma(\pi, \sigma)$ the diagram of a pair of partitions $(\pi, \sigma)$. For $n_{1}, n_{2}, \ldots, n_{k} \geq 1$, we write $n=\sum_{i=1}^{k} n_{i}$, and denote by $\pi^{*}$ the partition of $[n]$ given by

$$
\pi^{*}=\left\{\left\{1, \ldots, n_{1}\right\},\left\{n_{1}+1, \ldots, n_{1}+n_{2}\right\}, \ldots,\left\{n_{1}+\cdots+n_{k-1}+1, \ldots, n\right\}\right\} .
$$

We define the function in $n$ variables

$$
f_{1} \otimes_{0}, \cdots \otimes_{0} f_{k}=\prod_{i=1}^{k} f_{i}\left(x_{n_{1}+\cdots, n_{i-1}+1}, \ldots, x_{n_{1}+\cdots+n_{i}}\right), \quad n_{0}=0,
$$

and a set

$$
\mathcal{M}_{2}\left([n], \pi^{*}\right)=\left\{\sigma \in \mathcal{P}: \Gamma\left(\pi^{*}, \sigma\right) \text { is Gaussain, non-flat and connected }\right\} .
$$

Lemma 5. For any vector of functions $\left(f_{1}, \ldots, f_{k}\right)$ such that $f_{i} \in L_{s}^{2}(\mathcal{Z}, d \lambda), i=1, \ldots, k$, if $\mathcal{M}_{2}\left([n], \pi^{*}\right)=\varnothing$, then

$$
\kappa\left(I_{n_{1}}\left(f_{1}\right), \ldots, I_{n_{k}}\left(f_{k}\right)\right)=\sum_{\sigma \in \mathcal{M}_{2}\left([n], \pi^{*}\right)} \int_{\mathbb{R}^{n / 2}} f_{\sigma, k}(x) d \lambda(x),
$$

where the function $f_{\sigma, k}$ of $2 / n$ variables is obtained by identifying $x_{i}$ and $x_{j}$ in the argument of $f_{1} \otimes_{0}, \cdots \otimes_{0} f_{k}$ if and only if $i \sim_{\sigma} j$ (this notation means that $i$ and $j$ belong to the same block of $\sigma$ ).

Corollary 2. Under the assumptions in Theorem 2, we have that

(a) for $J=1$,

$$
\begin{aligned}
& \mathbb{P}\left(\frac{F}{G} \leq z\right)-\mathbb{P}(Z \leq z) \\
& =\frac{H_{1}(z) \phi(z)}{2(\mathbb{E}[G])^{2}}\left\{(\mathbb{E}[G])^{2}-2 \sum_{\sigma=\left(b_{1}, b_{2}\right) \in \mathcal{P}([2])} \int_{Z^{2}} \prod_{i \in b_{1}} f\left(z_{i}, z_{i+1}\right)\right. \\
& \left.\quad \times \prod_{i \in b_{2}}(-z)^{\left|b_{2}\right|} g\left(z_{i}, z_{i+1}\right) d v\left(z_{1}\right) v\left(d z_{2}\right)\right\}+R_{z}^{(2)}\left(A_{z}\right),
\end{aligned}
$$

where the remainder term $R_{z}^{(2)}\left(A_{z}\right)$ is given by (24). 
(b) for $J=2$,

$$
\begin{aligned}
& \mathbb{P}\left(\frac{F}{G} \leq z\right)-\mathbb{P}(Z \leq z) \frac{H_{1}(z) \phi(z)}{2(\mathbb{E}[G])^{2}}\left\{(\mathbb{E}[G])^{2}-2 \sum_{\sigma=\left(b_{1}, b_{2}\right) \in \mathcal{P}([2])} \int_{Z^{2}} \prod_{i \in b_{1}} f\left(z_{i}, z_{i+1}\right)\right. \\
&\left.\quad \times \prod_{i \in b_{2}}(-z)^{\left|b_{2}\right|} g\left(z_{i}, z_{i+1}\right) d v\left(z_{1}\right) v\left(d z_{2}\right)\right\} \\
&-\frac{8 H_{2}(z) \phi(z)}{(3 ! \mathbb{E}[G])^{3}} \sum_{\sigma=\left(b_{1}, b_{2}\right) \in \mathcal{P}([3])} \int_{Z^{3}} \prod_{i \in b_{1}} f\left(z_{i}, z_{i+1}\right) \\
& \quad \times \prod_{i \in b_{2}}(-z)^{\left|b_{2}\right|} g\left(z_{i}, z_{i+1}\right) d v\left(z_{1}\right) d v\left(z_{2}\right) v\left(d z_{3}\right) \\
&+R_{z}^{(3)}\left(A_{z}\right)
\end{aligned}
$$

where the remainder term $R_{z}^{(3)}\left(A_{z}\right)$ is given by (26).

Proof. Suppose that $\mathfrak{H}=L^{2}(Z, \mathcal{Z}, v)$, where $v$ is a $\sigma$-finite and non-atomic measure. By using Lemma 5, we obtain

$$
\begin{aligned}
& \kappa_{n}\left(I_{2}(f+(-z) g)\right) \\
&=2^{n-1}(n-1) ! \int_{Z^{n}}(f+(-z) g)\left(z_{1}, z_{2}\right)(f+(-z) g)\left(z_{2}, z_{3}\right) \times \cdots \\
& \cdots \times(f+(-z) g)\left(z_{n-1}, z_{n}\right)(f+(-z) g)\left(z_{n}, z_{1}\right) \\
& \quad \times d v\left(z_{1}\right) \cdots v\left(d z_{n}\right) \\
&=2^{n-1}(n-1) ! \sum_{\sigma=\left(b_{1}, b_{2}\right) \in \mathcal{P}([n])} \int_{Z^{n}} \prod_{i \in b_{1}} f\left(z_{i}, z_{i+1}\right) \\
& \quad \times \prod_{i \in b_{2}}(-z)^{\left|b_{2}\right|} g\left(z_{i}, z_{i+1}\right) d v\left(z_{1}\right) \cdots v\left(d z_{n}\right),
\end{aligned}
$$

where $\prod_{i \in b_{k}}=1$ if $b_{k}=\varnothing$ and $z_{n+1}=z_{1}$. Since $A_{z}=I_{2}(f+(-z) g) / \mathbb{E}[G]$, the result follows from (33).

\section{Optimal Berry-Esseen Bound}

In this section, the main result in this paper will be obtained. Theorem 5 below is an interesting result in itself because it is an extension of the optimal bound (4) in the introduction, in some respect. We consider sequences $F_{n}=I_{q}\left(f_{n}\right)$ and $\bar{G}_{n}=I_{q}\left(g_{n}\right), n \geq 1$, living in the $q$ th Wiener chaos, and such that each $F_{n}$ has the unit variance. In this case, we derive the optimal Berry-Esseen bound by using the two-term Edgeworth expansion given in Corollary 1. The following result provides some bounds on Gamma operators $\Gamma_{j}$.

Proposition 2. Let $F=I_{q}(f)$, where $f \in \mathfrak{H}^{\otimes q}$, with $\mathbb{E}\left[F^{2}\right]=1$. Then we have

$$
\begin{array}{ll}
\text { (a) } & \mathbb{E}\left[\left|1-\Gamma_{1}(F)\right|^{2}\right] \leq C_{q} \kappa_{4}(F), \\
\text { (b) } & \left.\mathbb{E}\left[\mid\left(1-\Gamma_{1}(F)\right) \Gamma_{2}^{*}(F)\right] \mid\right] \leq C_{q} \sqrt{\kappa_{4}(F)}\left(\left|\kappa_{3}(F)\right|+\kappa_{4}(F)^{3 / 4}\right), \\
\text { (c) } & \mathbb{E}\left[\left|\Gamma_{3}^{*}(F)\right|\right] \leq C_{q} \kappa_{4}(F) .
\end{array}
$$

Proof. By using Proposotion 4.3 in [10], it is immediate to get results. 
By regulating the remainder term in the expansion (15) of Theorem 2, the upper and lower bounds of the Kolmogorov distance between $F_{n} / G_{n}$ and $Z$ will be obtained. In this section, we assume that

$$
\begin{array}{ll}
\left(\mathrm{A}_{1}\right) & \lim _{n \rightarrow \infty} \kappa_{2 e_{2}}\left(X_{n}\right)=0, \\
\left(\mathrm{~A}_{2}\right) & \lim _{n \rightarrow \infty} \kappa_{(3-\alpha) e_{1}+\alpha e_{2}}\left(X_{n}\right)=0 \alpha=1,2,3, \\
\left(\mathrm{~A}_{3}\right) & \lim _{n \rightarrow \infty} \kappa_{(4-\alpha) e_{1}+\alpha e_{2}}\left(X_{n}\right)=0 \alpha=0,1,2,3,4 .
\end{array}
$$

Theorem 3 (Upper bounds). Let $F_{n}=I_{q}\left(f_{n}\right)$ and $\bar{G}_{n}=I_{q}\left(g_{n}\right)$ for all $q \geq 2$, where $\bar{G}_{n}=$ $G_{n}-\mathbb{E}\left[G_{n}\right]$, and let $X_{n}=\left(F_{n}, \bar{G}_{n}\right)$ such that $\mathbb{E}\left[G_{n}\right]=\kappa_{2 e_{1}}\left(X_{n}\right)<\infty$. Assume that there exists a positive sequence $\left\{a_{n}\right\}$ such that $a_{n} \rightarrow \infty$, and

$$
\begin{aligned}
& \text { (i) } \quad \sup _{n} \frac{\left|\kappa_{2 e_{2}}\left(X_{n}\right)\right|}{\left|\kappa_{e_{1}+e_{2}}\left(X_{n}\right)\right|}<\infty, \\
& \text { (ii) } \quad \sup _{n} \frac{a_{n}^{\alpha}\left|\kappa_{(3-\alpha) e_{1}+\alpha e_{2}}\left(X_{n}\right)\right|}{\left|\kappa_{3 e_{1}}\left(X_{n}\right)\right|}<\infty, \alpha=1,2,3, \\
& \text { (iii) } \quad \sup _{n} \frac{a_{n}^{\alpha}\left|\kappa_{(4-\alpha) e_{1}+\alpha e_{2}}\left(X_{n}\right)\right|^{3 / 4}}{\left|\kappa_{3 e_{1}}\left(X_{n}\right)\right|}<\infty, \alpha=0,1,2,3,4 .
\end{aligned}
$$

Assume that

$$
\sup _{n} \frac{\bar{\Phi}\left(a_{n}\right)}{M_{n}}<\infty,
$$

where $\bar{\Phi}(x)=1-\Phi(x)$, and

$$
M_{n}=\max \left\{\frac{\left|\kappa_{e_{1}+e_{2}}\left(X_{n}\right)\right|}{\left(\mathbb{E}\left[G_{n}\right]\right)^{2}}, \frac{\left|\kappa_{3 e_{1}}\left(X_{n}\right)\right|}{\left(\mathbb{E}\left[G_{n}\right]\right)^{3}}\right\} .
$$

Then there exists a constant $C>0$ such that

$$
\sup _{z \in \mathbb{R}}\left|\mathbb{P}\left(\frac{F_{n}}{G_{n}} \leq z\right)-\mathbb{P}(Z \leq z)\right| \leq C \max \left\{M_{n} \frac{\left|\left(\mathbb{E}\left[G_{n}\right]\right)^{2}-\kappa_{2 e_{1}}\left(X_{n}\right)\right|}{\left(\mathbb{E}\left[G_{n}\right]\right)^{2}}\right\} .
$$

Proof. Obviously, it is sufficient to consider $z \geq 0$ to prove this theorem. From (23) of Corollary 1 , one can deduce that

$$
\begin{aligned}
& \left|\mathbb{P}\left(\frac{F_{n}}{G_{n}} \leq z\right)-\mathbb{P}(Z \leq z)\right| \\
\leq & \frac{\left|H_{1}(z)\right| \phi(z)}{2\left(\mathbb{E}\left[G_{n}\right]\right)^{2}}\left\{\left|\left(\mathbb{E}\left[G_{n}\right]\right)^{2}-\kappa_{2 e_{1}}\left(X_{n}\right)\right|+2|z|\left|\kappa_{e_{1}+e_{2}}\left(X_{n}\right)\right|\right. \\
& \left.+z^{2}\left|\kappa_{2 e_{2}}\left(X_{n}\right)\right|\right\}+\left|R_{z}^{(2)}\left(A_{z, n}\right)\right| \\
\leq & \frac{C}{\left(\mathbb{E}\left[G_{n}\right]\right)^{2}}\left\{\left|\left(\mathbb{E}\left[G_{n}\right]\right)^{2}-\kappa_{2 e_{1}}\left(X_{n}\right)\right|+\left|\kappa_{e_{1}+e_{2}}\left(X_{n}\right)\right|\right. \\
& \left.+\left|\kappa_{2 e_{2}}\left(X_{n}\right)\right|\right\}+\left|R_{z}^{(2)}\left(A_{z, n}\right)\right|,
\end{aligned}
$$

where

$$
A_{z, n}=\frac{(1,-z) \cdot X_{n}}{\mathbb{E}\left[G_{n}\right]}
$$


Now the remainder term $R_{z}^{(2)}\left(A_{z, n}\right)$ in (39) will be estimated. By using Lemma 1 and Proposition 2, the first expectation in (24) can be bounded as follows:

$$
\begin{aligned}
& \left|\mathbb{E}\left[\Psi_{1} f_{z}^{\prime}\left(A_{z, n}\right) \bar{\Gamma}_{1}\left(A_{z, n}\right)^{2}\right]\right| \\
\leq & \left\|\Psi_{1} f_{z}^{\prime}\right\|_{\infty} \mathbb{E}\left[\bar{\Gamma}_{1}\left(A_{z, n}\right)^{2}\right] \\
\leq & \frac{C}{\left(\mathbb{E}\left[G_{n}\right]\right)^{4}} \sum_{\alpha=0}^{4}|z|^{\alpha}\left|\mathcal{K}_{(4-\alpha) e_{1}+\alpha_{2}}\left(X_{n}\right)\right| .
\end{aligned}
$$

Similarly, the second term in the remainder (24) can be estimated as

$$
\begin{aligned}
& \left|\mathbb{E}\left[\Psi_{0} f_{z}^{\prime}\left(A_{z, n}\right) \Gamma_{2}^{*}\left(A_{z . n}\right)\right]\right| \\
\leq & 2\left\|\Psi_{0} f_{z}^{\prime}\right\|_{\infty} \sqrt{\mathbb{E}\left[\Gamma_{2}^{*}\left(A_{z, n}\right)^{2}\right]} \\
\leq & \frac{C}{\left|\mathbb{E}\left[G_{n}\right]\right|^{3}} \sum_{\alpha=0}^{3}|z|^{\alpha}\left|\kappa_{(3-\alpha) e_{1}+\alpha e_{2}}\left(X_{n}\right)\right| \\
& +\frac{C}{\left(\mathbb{E}\left[G_{n}\right]\right)^{3}} \sum_{\alpha=0}^{4}|z|^{3 \alpha / 4}\left|\kappa_{(4-\alpha) e_{1}+\alpha_{2}}\left(X_{n}\right)\right|^{3 / 4} .
\end{aligned}
$$

Combining (40) and (41), one can see that the right-hand side of (39) can be bounded as

$$
\begin{aligned}
& \left|\mathbb{P}\left(\frac{F_{n}}{G_{n}} \leq z\right)-\mathbb{P}(Z \leq z)\right| \\
\leq & \frac{C}{\left.\mathbb{E}\left[G_{n}\right]\right)^{2}}\left\{\left|\left(\mathbb{E}\left[G_{n}\right]\right)^{2}-\kappa_{2 e_{1}}\left(X_{n}\right)\right|+\left|\kappa_{e_{1}+e_{2}}\left(X_{n}\right)\right|+\left|\kappa_{2 e_{2}}\left(X_{n}\right)\right|\right\} \\
& +\frac{C}{\left|\mathbb{E}\left[G_{n}\right]\right|^{3}} \sum_{\alpha=0}^{3}|z|^{\alpha}\left|\kappa_{(3-\alpha) e_{1}+\alpha e_{2}}\left(X_{n}\right)\right| \\
& +\frac{C}{\left(\mathbb{E}\left[G_{n}\right]\right)^{3}} \sum_{\alpha=0}^{4}\left(|z|^{3 \alpha / 4} \vee|z|^{\alpha}\right)\left|\kappa_{(4-\alpha) e_{1}+\alpha_{2}}\left(X_{n}\right)\right|^{3 / 4} .
\end{aligned}
$$

From (42), it follows that, for sufficiently large $n$,

$$
\begin{aligned}
& \sup _{z \leq a_{n}}\left|\mathbb{P}\left(\frac{F_{n}}{G_{n}} \leq z\right)-\mathbb{P}(Z \leq z)\right| \\
\leq & \frac{C}{\left(\mathbb{E}\left[G_{n}\right]\right)^{2}}\left[\left|\left(\mathbb{E}\left[G_{n}\right]\right)^{2}-\kappa_{2 e_{1}}\left(X_{n}\right)\right|+\left|\kappa_{e_{1}+e_{2}}\left(X_{n}\right)\right|\left\{1+\sup _{n} \frac{\left|\kappa_{2 e_{2}}\left(X_{n}\right)\right|}{\left|\kappa_{e_{1}+e_{2}}\left(X_{n}\right)\right|}\right\}\right] \\
+ & \frac{C\left|\kappa_{3 e_{1}}\left(X_{n}\right)\right|}{\left|\mathbb{E}\left[G_{n}\right]\right|^{3}}\left\{\sup _{n} \frac{\sum_{\alpha=1}^{3} a_{n}^{\alpha}\left|\kappa_{(3-\alpha) e_{1}+\alpha e_{2}}\left(X_{n}\right)\right|}{\left|\kappa_{3 e_{1}}\left(X_{n}\right)\right|}\right. \\
& \left.\quad \sup _{n} \frac{\sum_{\alpha=0}^{4} a_{n}^{\alpha}\left|\kappa_{(4-\alpha) e_{1}+\alpha e_{2}}\left(X_{n}\right)\right|^{3 / 4}}{\left|\kappa_{3 e_{1}}\left(X_{n}\right)\right|}\right\} \\
\leq & C \max \left\{M_{n}, \frac{\left|\left(\mathbb{E}\left[G_{n}\right]\right)^{2}-\kappa_{2 e_{1}}\left(X_{n}\right)\right|}{\left(\mathbb{E}\left[G_{n}\right]\right)^{2}}\right\} .
\end{aligned}
$$


On the other hand, it follows, from (43), that

$$
\begin{aligned}
& \sup _{z>a_{n}}\left|\mathbb{P}\left(\frac{F_{n}}{G_{n}} \leq z\right)-\mathbb{P}(Z \leq z)\right| \\
\leq & \left|\mathbb{P}\left(\frac{F_{n}}{G_{n}} \leq a_{n}\right)-\mathbb{P}\left(Z \leq a_{n}\right)\right|+2 \bar{\Phi}\left(a_{n}\right) \\
\leq & C \max \left\{M_{n}, \frac{\left|\left(\mathbb{E}\left[G_{n}\right]\right)^{2}-\kappa_{2 e_{1}}\left(X_{n}\right)\right|}{\left(\mathbb{E}\left[G_{n}\right]\right)^{2}}\right\} .
\end{aligned}
$$

Combining (43) and (44) completes the proof of this theorem.

Theorem 4 (Lower bounds). Let $F_{n}=I_{q}\left(f_{n}\right)$ and $\bar{G}_{n}=I_{q}\left(g_{n}\right)$ for all $q \geq 2$, where $\bar{G}_{n}=$ $G_{n}-\mathbb{E}\left[G_{n}\right]$ and $\mathbb{E}\left[G_{n}\right]=\kappa_{2 e_{1}}\left(X_{n}\right)$, and let $X_{n}=\left(F_{n}, \bar{G}_{n}\right)$. Suppose that

$$
\begin{aligned}
& \text { (i) } \lim _{n \rightarrow \infty} \frac{\sum_{\alpha=0}^{4}\left|\kappa_{(4-\alpha) e_{1}+\alpha e_{2}}\left(X_{n}\right)\right|}{\left(\mathbb{E}\left[G_{n}\right]\right)^{2}\left|\kappa_{e_{1}+e_{2}}\left(X_{n}\right)\right|}=0, \\
& \text { (ii) } \lim _{n \rightarrow \infty} \frac{\sqrt{\sum_{\alpha=0}^{4}\left|\kappa_{(4-\alpha) e_{1}+\alpha e_{2}}\left(X_{n}\right)\right|} \sum_{\alpha=0}^{3}\left|\kappa_{(3-\alpha) e_{1}+\alpha e_{2}}\left(X_{n}\right)\right|}{\mathbb{E}\left[G_{n}\right]\left|\kappa_{e_{1}+e_{2}}\left(X_{n}\right)\right|}=0, \\
& \text { (iii) } \lim _{n \rightarrow \infty} \frac{\kappa_{4 e_{1}}\left(X_{n}\right)}{\mathbb{E}\left[G_{n}\right]\left|\kappa_{3 e_{1}}\left(X_{n}\right)\right|}=0 .
\end{aligned}
$$

Then there exists a constant $C>0$ such that

$$
\sup _{z \in \mathbb{R}}\left|\mathbb{P}\left(\frac{F_{n}}{G_{n}} \leq z\right)-\mathbb{P}(Z \leq z)\right| \geq C M_{n}
$$

Proof. Since $H_{2}(z)=0$ for $z=1$ and $z=-1$, we have, from (25), that

$$
\begin{aligned}
& \mathbb{P}\left(\frac{F_{n}}{G_{n}} \leq 1\right)-\mathbb{P}(Z \leq 1) \\
= & \frac{\phi(1)}{2\left(\mathbb{E}\left[G_{n}\right]\right)^{2}}\left\{\kappa_{e_{1}+e_{2}}\left(X_{n}\right)-\kappa_{2 e_{2}}\left(X_{n}\right)\right\}+R_{1}^{(3)}\left(A_{1, n}\right),
\end{aligned}
$$

and

$$
\begin{aligned}
& \mathbb{P}\left(\frac{F_{n}}{G_{n}} \leq-1\right)-\mathbb{P}(Z \leq-1) \\
= & \frac{\phi(-1)}{2\left(\mathbb{E}\left[G_{n}\right]\right)^{2}}\left\{-\kappa_{e_{1}+e_{2}}\left(X_{n}\right)-\kappa_{2 e_{2}}\left(X_{n}\right)\right\}+R_{-1}^{(3)}\left(A_{-1, n}\right) .
\end{aligned}
$$

Substracting (50) from (49) yields that

$$
\begin{aligned}
& \left\{\mathbb{P}\left(\frac{F_{n}}{G_{n}} \leq 1\right)-\mathbb{P}(Z \leq 1)\right\}-\left\{\mathbb{P}\left(\frac{F_{n}}{G_{n}} \leq-1\right)-\mathbb{P}(Z \leq-1)\right\} \\
= & \frac{\phi(1)}{\left(\mathbb{E}\left[G_{n}\right]\right)^{2}} \kappa_{e_{1}+e_{2}}\left(X_{n}\right)+R_{1}^{(3)}\left(A_{1, n}\right)-R_{-1}^{(3)}\left(A_{-1, n}\right) .
\end{aligned}
$$


By using Proposition 2, together with (51), we have that

$$
\begin{aligned}
& \sup _{z \in \mathbb{R}}\left|\mathbb{P}\left(\frac{F_{n}}{G_{n}} \leq z\right)-\mathbb{P}(Z \leq z)\right| \\
\geq & \left|\left\{\mathbb{P}\left(\frac{F_{n}}{G_{n}} \leq 1\right)-\mathbb{P}(Z \leq 1)\right\}-\left\{\mathbb{P}\left(\frac{F_{n}}{G_{n}} \leq-1\right)-\mathbb{P}(Z \leq-1)\right\}\right| \\
\geq & \frac{\phi(1)}{\left(\mathbb{E}\left[G_{n}\right]\right)^{2}}\left|\kappa_{e_{1}+e_{2}}\left(X_{n}\right)\right|\left\{1-c\left(\frac{\sum_{\alpha=0}^{4}\left|\kappa_{(4-\alpha) e_{1}+\alpha e_{2}}\left(X_{n}\right)\right|}{\left(\mathbb{E}\left[G_{n}\right]\right)^{2}\left|\kappa_{e_{1}+e_{2}}\left(X_{n}\right)\right|}\right.\right. \\
& \left.\left.+\frac{\sqrt{\sum_{\alpha=0}^{4}\left|\kappa_{(4-\alpha) e_{1}+\alpha e_{2}}\left(X_{n}\right)\right|} \sum_{\alpha=0}^{3}\left|\kappa_{(3-\alpha) e_{1}+\alpha e_{2}}\left(X_{n}\right)\right|}{\left(\mathbb{E}\left[G_{n}\right]\right)^{2}\left|\kappa_{e_{1}+e_{2}}\left(X_{n}\right)\right|}\right)\right\} .
\end{aligned}
$$

On the other hand, since $H_{1}(z)=0$ for $z=0$, one immediately see, from the case of $J=2$ in Corollary 1, that

$$
\mathbb{P}\left(\frac{F_{n}}{G_{n}} \leq 0\right)-\mathbb{P}(Z \leq 0)=\frac{\phi(0)}{3 !\left(\mathbb{E}\left[G_{n}\right]\right)^{3}} \kappa_{3 e_{1}}\left(X_{n}\right)+R_{0}^{(3)}\left(A_{1, n}\right) .
$$

By a similar estimate as for (52), we obtain

$$
\begin{aligned}
& \sup _{z \in \mathbb{R}}\left|\mathbb{P}\left(\frac{F_{n}}{G_{n}} \leq 0\right)-\mathbb{P}(Z \leq 0)\right| \\
& \geq \frac{\phi(0)}{3 !\left|\mathbb{E}\left[G_{n}\right]\right|^{3}}\left|\kappa_{3 e_{1}}\left(X_{n}\right)\right|-\left|R_{0}^{(3)}\left(A_{1, n}\right)\right| \\
& \geq \frac{\phi(0)}{3 !\left|\mathbb{E}\left[G_{n}\right]\right|^{3}}\left|\kappa_{3 e_{1}}\left(X_{n}\right)\right|\left\{1-c\left(\frac{\kappa_{4 e_{1}}\left(X_{n}\right)}{\mathbb{E}\left[G_{n}\right]\left|\kappa_{3 e_{1}}\left(X_{n}\right)\right|}\right.\right. \\
&\left.\left.\left.+\mathbb{E}\left[G_{n}\right]\right)^{2} \sqrt{\kappa_{4 e_{1}}\left(X_{n}\right)}\right)\right\} .
\end{aligned}
$$

Combining (52) and (54) yields the lower bound (48).

By using Theorems 3 and 4, we get an optimal bound for the normal approxiamtion of $F_{n} / G_{n}$.

Theorem 5 (Optimal bound). Let $F_{n}=I_{q}\left(f_{n}\right)$ and $\bar{G}_{n}=I_{q}\left(g_{n}\right)$ for all $q \geq 2$, where $\bar{G}_{n}=$ $G_{n}-\mathbb{E}\left[G_{n}\right]$ and $\mathbb{E}\left[G_{n}\right]=\kappa_{2 e_{1}}\left(X_{n}\right)$, and let $X_{n}=\left(F_{n}, \bar{G}_{n}\right)$. Suppose that the assumptions (i), (ii) and (iii) in Theorems 3 and 4 are satisfied. Moreover, assume that

$$
\sup _{n} \frac{\bar{\Phi}\left(a_{n}\right)}{M_{n}}<\infty
$$

and

$$
\sup _{n} \frac{\left|\left(\mathbb{E}\left[G_{n}\right]\right)^{2}-\kappa_{2 e_{1}}\left(X_{n}\right)\right|}{\left(\mathbb{E}\left[G_{n}\right]\right)^{2} M_{n}}<\infty .
$$

Then there exist constants $0<c<C<\infty$ such that

$$
c M_{n} \leq \sup _{z \in \mathbb{R}}\left|\mathbb{P}\left(\frac{F_{n}}{G_{n}} \leq z\right)-\mathbb{P}(Z \leq z)\right| \leq C M_{n} .
$$




\section{Applications}

In this section, we apply Theorem 5 to the estimation problem of parameters appearing in SDEs and SPDEs considered in previous works $[12,19,20]$.

\subsection{Stochastic Partial Differential Equation}

In [21], the authors investigate asymptotic properties of the MLE for parameters occurring in parabolic SPDEs associated with the operator $A^{\theta} \equiv \theta A_{1}+A_{0}$, and driven by a cylindrical Brownian motion $W$ :

$$
\begin{aligned}
\frac{\partial u(t, x)}{\partial t} & =A^{\theta}(x) u(t, x)+\frac{\partial}{\partial t} W(t, x), \\
u(0, x) & =u_{0}(x), \\
\left.D^{\gamma} u(t, x)\right|_{\partial G} & =0 \text { for all multi-indices } \gamma \text { such that }|\gamma| \leq m-1,
\end{aligned}
$$

where $A_{0}$ and $A_{1}$ are partial differential operators of orders $m_{0}$ and $m_{1}$, so that $\left\{A^{\theta}: \theta \in \Theta\right\}$ are operators of order $2 m=\max \left(m_{1}, m_{0}\right)$. Problem (58)-(60) is understood in the sense of distributions. Some basic assumptions and definitions are described in Section 2 of the paper [21]. For the sake of completeness, we will briefly review these things. The main assumption is that the Equation (58) is diagonalizable. A complete orthonormal (in $L^{2}(G)$ ) system $\left\{h_{i}\right\}_{i=1}^{\infty}$ is a common system of eigenfunction of the operators $A_{0}$ and $A_{1}$;

$$
A_{0} h_{i}=k_{i} h_{i}, A_{1} h_{i}=v_{i} h_{i},
$$

and hence $A^{\theta} h_{i}=\mu_{i}^{\theta} h_{i}$, where $\mu_{i}^{\theta}=\theta v_{i}+k_{i}$. For $s \geq 0$, set

$$
\mathbb{H}^{s}=\left\{u \in L^{2}(G):\|u\|_{s} \equiv\left(\sum_{j=1}^{\infty} \lambda_{j}^{2 s}\left|\left\langle u, h_{j}\right\rangle_{L_{2}(G)}\right|^{2}\right)^{1 / 2}<\infty\right\} .
$$

For $s<0, \mathbb{H}^{s}$ is a closure of $L_{2}(G)$ in the norm $\|u\|_{s}$ defined for $s<0$ by the same formula as for $s>0$. For $s \in \mathbb{R}, \mathbb{H}^{s}$ is a Hilbert space with respect to the inner product $\langle\cdot, \cdot\rangle_{s}$ associated with the norm $\|u\|_{s}$ and the functions $h_{i}^{s}=\lambda_{i}^{-s} h_{i}, i=1,2, \ldots$, form an orthonormal basis in $\mathbb{H}^{s}$.

Proposition 3 (Huebner and Rozovskii). For fixed $\alpha>d / 2$ and $\theta \in \Theta$, assume that $u_{0} \in \mathbb{H}^{-\alpha}$. Then $u^{\theta}(t, x)=\sum_{i=1}^{\infty} u_{i}^{\theta}(t) h_{i, \theta}^{-\alpha}(x)$ is the only solution of problem (58)-(60), where the Fourier coefficient $u_{i}^{\theta}(t)$ is a solution of the one-dimensional Ornstein-Uhlenbeck equation

$$
\left\{\begin{aligned}
d u_{i}^{\theta}(t) & =\mu_{i}^{\theta} u_{i}^{\theta}(t) d t+\lambda_{i, \theta}^{-\alpha} d W_{i}(t) \\
u_{i}^{\theta}(0) & =\left\langle u_{0}, h_{i, \theta}^{-\alpha}\right\rangle_{-\alpha}
\end{aligned}\right.
$$

where $W_{i}(t), i=1,2, \ldots$, are independent one-dimensional Wiener processes.

The projection of the solution $u(t, x)$ onto the subspace spanned by $\left\{h_{1}^{-\alpha}, \ldots, h_{N}^{-\alpha}\right\}$ is given by

$$
u^{N}(t, x) \equiv \sum_{i=1}^{N} u_{i}(t) h_{i}^{-\alpha}(x) .
$$

Let $\mathcal{B}_{T}^{N}$ be the Borel $\sigma$-algebra on $C\left([0, T] ; \mathbb{R}^{N}\right)$, and $P_{\theta}^{N}$ be the measure on $\mathcal{B}_{T}^{N}$ generated by $u^{N, \theta}$. The MLE $\hat{\theta}^{N}$ corresponding to the likelihood ratio $\frac{d P_{\theta}^{N}}{d P_{\theta_{0}}^{N}}\left(u^{N}\right)$ is given by

$$
\hat{\theta}^{N}=\theta_{0}+\frac{\int_{0}^{T}\left\langle A_{1} u^{N}(t), d W^{N}(t)\right\rangle_{0}}{\int_{0}^{T}\left\|A_{1} u^{N}(t)\right\|_{0}^{2} d t} .
$$


Let $\mathcal{N}$ be an uniformly elliptic operator of the order $m$. Then define

$$
\zeta_{\mathcal{N}}=\left(\frac{1}{2 \pi} \int_{P_{\mathcal{N}}(x, \xi)<1} d x d \xi\right)^{-m / d},
$$

where $P_{\mathcal{N}}(x, \xi)$ is the principal symbol of the operator $\mathcal{N}$. In [21], the authors prove the problem of asymptotic normality of the MLE $\hat{\theta}^{N}$.

Theorem 6 (Huebner and Rozovskii). Assume that $m_{1} \geq m-\frac{d}{2}$ and set

$$
\Psi_{N}= \begin{cases}\frac{\zeta}{4 \tau} T N^{2 \tau}+o\left(N^{2 \tau}\right) & \text { if } m_{1}>m-d / 2, \\ \frac{\zeta}{2} T \ln N+o(\ln N) & \text { if } m_{1}=m-d / 2,\end{cases}
$$

where $\tau=\left(m_{1}-m\right) / d+1 / 2$ and

$$
\zeta=\frac{\zeta_{A^{\theta}}^{2 m / d}}{\zeta_{A_{1}}^{2 m_{1} / d}}
$$

Then $\sqrt{\Psi_{N}}\left(\hat{\theta}^{N}-\theta\right)$ converges in distribution to a Gaussian random variable with zero mean and unit variance as $N \uparrow \infty$.

Obviously, the Fisher Information $I_{N}(\theta)$ is given by

$$
I_{N}(\theta)=\sum_{i=1}^{N} \frac{v_{i}^{2}}{2 \mu_{i}}\left|\lambda_{i}^{\alpha} u_{i}(0)\right|^{2}\left(e^{2 \mu_{i} T}-1\right)-\frac{T}{2} \sum_{i=1}^{N} \frac{v_{i}^{2}}{\mu_{i}}+\sum_{i=1}^{N} \frac{v_{i}^{2}}{4 \mu_{i}^{2}}\left(e^{2 \mu_{i} T}-1\right) .
$$

Since $u_{0} \in L^{2}(G)$, we have $\left\langle u_{0}, h_{n}\right\rangle_{0}^{2}=\lambda_{i}^{2 \alpha}\left\langle u_{0}, h_{n}^{-\alpha}\right\rangle_{-\alpha}^{2}=\lambda_{i}^{2 \alpha} u_{i}(0) \rightarrow 0$ as $n \rightarrow \infty$. So the second sum in (65) is the main term of $I_{N}(\theta)$. This implies that we may assume $u_{0}=0$ to find the rate of convergence of the distribution of $\sqrt{I_{N}(\theta)}\left(\hat{\theta}^{N}-\theta_{0}\right)$. Define the kernels $f_{2}^{(i)}$ and $g_{2}^{(i)}$ for $i=1, \ldots, N$ as

$$
\begin{aligned}
& f_{2}^{(i)}(s, t)=v_{i} e^{\mu_{i}|t-s|}, \\
& g_{2}^{(i)}(s, t)=\frac{v_{i}^{2}}{2 \mu_{i}} e^{-\mu_{i}(s+t)}\left(e^{2 \mu_{i} T}-e^{2 \mu_{i}(s \vee t)}\right) .
\end{aligned}
$$

By Lemma 1 in [12], we can write

$$
\sqrt{I_{N}(\theta)}\left(\hat{\theta}_{N}-\theta\right)=\frac{F_{N}}{\bar{G}_{N}+1}
$$

where

$$
F_{N}=\frac{\sum_{i=1}^{N} I_{2}\left(f_{2}^{(i)}\right)}{2 \sqrt{I_{N}(\theta)}} \text { and } \bar{G}_{N}=\frac{\sum_{i=1}^{N} I_{2}\left(g_{2}^{(i)}\right)}{I_{N}(\theta)} .
$$

Then we write $X_{N}=\left(F_{N}, \bar{G}_{N}\right)$.

Lemma 6. Assume that $m_{1}>m-\frac{d}{2}$. Then there exists a constant $c=c\left(T, \theta, m, m_{1}, d\right)$, depending on $T, \theta, m, m_{1}$ and $d$ such that, for sufficiently large $N \in \mathbb{N}$,

$$
\kappa_{2 e_{1}}\left(X_{N}\right)=1,
$$


and for $\alpha=0,1, \ldots, \beta$, where $\beta=2,3,4$,

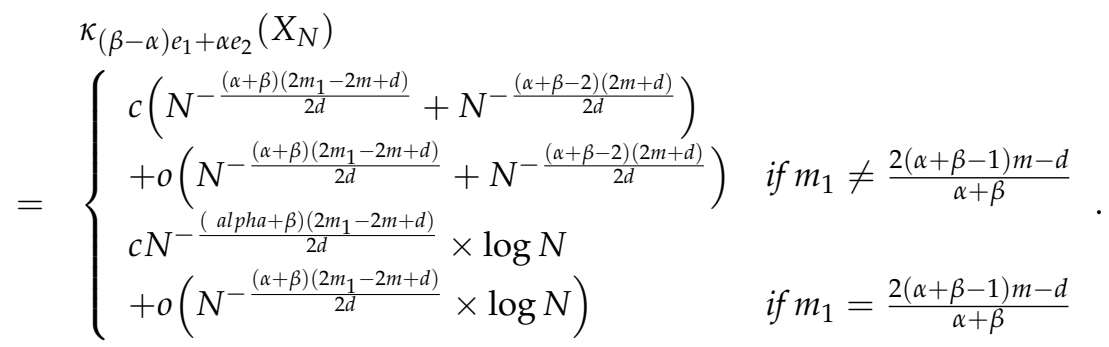

Proof. Direct computations yield that

$$
\begin{aligned}
\kappa_{2 e_{1}}\left(X_{N}\right) & =\frac{1}{2 I_{N}(\theta)} \sum_{i=1}^{N} \int_{0}^{T} \int_{0}^{T} f^{(i)}\left(z_{1}, z_{2}\right)^{2} d z_{1} d z_{2} \\
& =1
\end{aligned}
$$

Using $e^{\mu_{i}|t-s|}=e^{-\mu_{i}(s+t)} e^{2 \mu_{i}(s \vee t)}$, the function $g_{2}^{(i)}$ in (66) can be written as

$$
\begin{aligned}
g_{2}^{(i)}(s, t) & =\frac{v_{i}^{2}}{2 \mu_{i}} e^{-\mu_{i}(s+t)} e^{2 \mu_{i} T}-\frac{v_{i}}{2 \mu_{i}} f_{2}^{(i)}(s, t) \\
& =-\frac{v_{i}^{2}}{2 \mu_{i}}\left(e^{\mu_{i}|t-s|}-e^{-\mu_{i}(s+t)} e^{2 \mu_{i} T}\right) .
\end{aligned}
$$

Since $e^{2 \mu_{i} T} e^{-\mu_{i}(s+t)} \leq e^{\mu_{i}|s-t|}$ for all $(s, t) \in[0, T]^{2}$, it is sufficient, from (71), to consider the term $\frac{v_{i}}{2 \mu_{i}} f_{2}^{(i)}(s, t)$ in $g_{2}^{(i)}(s, t)$ as the main term of the asymptotics of $\kappa_{(\beta-\alpha) e_{1}+\alpha e_{2}}\left(X_{N}\right)$ for $\alpha=0,1, \ldots, \beta$, where $\beta=2,3,4$. Therefore, the main term is given as follows:

$$
\begin{aligned}
& Q_{N}(\alpha, \beta) \\
= & \frac{(-1)^{\alpha}}{I_{N}(\theta)^{\frac{\beta+\alpha}{2}}} \sum_{i=1}^{N} \frac{v_{i}^{\beta+\alpha}}{\mu_{i}^{\alpha}} \int_{[0, T]^{\beta}} \prod_{i=1}^{\beta} e^{\mu_{i}\left|z_{i}-z_{i+1}\right|} d z_{1} \ldots d z_{\beta} \\
= & \frac{\beta !(-1)^{\alpha}}{I_{N}(\theta)^{\frac{\beta+\alpha}{2}}} \sum_{i=1}^{N} \frac{v_{i}^{\beta+\alpha}}{\mu_{i}^{\alpha}} \int_{\left\{0 \leq z_{1} \leq \ldots \leq z_{\beta} \leq T\right\}} e^{2 \mu_{i}\left(z_{\beta}-z_{1}\right)} d z_{1} \ldots d z_{4} \\
= & \frac{4 !(-1)^{\alpha}}{2^{\alpha+\beta-1} I_{N}(\theta)^{\frac{\beta+\alpha}{2}}} \sum_{i=1}^{N} \frac{v_{i}^{\beta+\alpha}}{\mu_{i}^{\alpha+\beta-1}}+o\left(\frac{1}{I_{N}(\theta)^{\frac{\beta+\alpha}{2}}} \sum_{i=1}^{N} \frac{v_{i}^{\beta+\alpha}}{\mu_{i}^{\alpha+\beta-1}}\right) .
\end{aligned}
$$

Here we define $z_{\beta+1}=z_{1}$. From (72), it follows that

$$
\begin{aligned}
& \kappa_{(\beta-\alpha) e_{1}+\alpha e_{2}}\left(X_{N}\right) \\
& =Q_{N}(\alpha, \beta)+o\left(Q_{N}(\alpha, \beta)\right) \\
& =\left\{\begin{array}{ll}
c\left(N^{-\frac{(\alpha+\beta)\left(2 m_{1}-2 m+d\right)}{2 d}}+N^{\left.-\frac{(\alpha+\beta-2)(2 m+d)}{2 d}\right)}\right. & \\
+o\left(N^{-\frac{(\alpha+\beta)\left(2 m_{1}-2 m+d\right)}{2 d}}+N^{\left.-\frac{(\alpha+\beta-2)(2 m+d)}{2 d}\right)}\right) & \text { if } m_{1} \neq \frac{2(\alpha+\beta-1) m-d}{\alpha+\beta} \\
c N^{-\frac{(\alpha+\beta)\left(2 m_{1}-2 m+d\right)}{2 d} \times \log N} & \text { if } m_{1}=\frac{2(\alpha+\beta-1) m-d}{\alpha+\beta} \\
+o\left(N^{-\frac{(\alpha+\beta)\left(2 m_{1}-2 m+d\right)}{2 d}} \times \log N\right) & .
\end{array} .\right.
\end{aligned}
$$

Hence the proof of this lemma is completed.

Now we derive an optimal Berry-Esseen bound for the MLE $\hat{\theta}_{N}$. 
Theorem 7 (Optimal bound). Assume that $m_{1} \geq \frac{4 m}{3}-\frac{d}{3}$. Then there exist constants $0<$ $c_{T, \theta}<C_{T, \theta}<\infty$, depending on $\theta$ and $T$, such that, for sufficiently large $N \in \mathbb{N}$,

$$
\begin{gathered}
c_{T, \theta} N^{-\frac{2 m+d}{2 d}} \leq \sup _{z \in \mathbb{R}}\left|\mathbb{P}\left(\sqrt{I_{N}(\theta)}\left(\hat{\theta}_{N}-\theta\right) \leq z\right)-\mathbb{P}(Z \leq z)\right| \\
\leq C_{T, \theta} N^{-\frac{2 m+d}{2 d}} .
\end{gathered}
$$

Proof. From Lemma 6, compare the orders $N^{-\frac{(\alpha+\beta)\left(2 m_{1}-2 m+d\right)}{2 d}}$ and $N^{-\frac{(\alpha+\beta-2)(2 m+d)}{2 d}}$. Obviously, for all $\alpha=0,1, \ldots, \beta$, where $\beta=2,3,4$ except in the case of $\alpha=0$ and $\beta=2$, we have that

$$
\begin{aligned}
& \frac{(\alpha+\beta)\left(2 m_{1}-2 m+d\right)}{2 d}-\frac{(\alpha+\beta-2)(2 m+d)}{2 d} \\
\geq & \frac{2(\alpha+\beta)}{2 d}\left[m_{1}-\left\{2 m-\min _{3 \leq \alpha+\beta \leq 8} \frac{1}{\alpha+\beta}(2 m+d)\right\}\right] \\
\geq & \frac{2(\alpha+\beta)}{2 d}\left\{m_{1}-\left(\frac{4 m}{3}-\frac{d}{3}\right)\right\},
\end{aligned}
$$

so that if

$$
m_{1} \geq \frac{4 m}{3}-\frac{d}{3}
$$

the main term in asymptotics of the cumulants is given by $N^{-\frac{(\alpha+\beta-2)(2 m+d)}{2 d}}$. It is clear, from (67), that $\mathbb{E}\left[G_{N}\right]=\kappa_{2 e_{1}}\left(X_{N}\right)=1$, which satisfies the condition (56) in Theorem 5 . Now we prove the conditions (i), (ii) and (iii) in Theorem 5. It is obvious, from Lemma 6, that

$$
\begin{aligned}
& \text { (i) } \quad \sup _{N} \frac{\left|\kappa_{2 e_{2}}\left(X_{N}\right)\right|}{\left|\kappa_{e_{1}+e_{2}}\left(X_{n}\right)\right|}=\sup _{N} N^{-\frac{2 m+d}{2 d}}=1, \\
& \text { (ii) } \quad \sup _{N} \frac{a_{N}^{\alpha}\left|\mathcal{\kappa}_{(3-\alpha) e_{1}+\alpha e_{2}}\left(X_{N}\right)\right|}{\left|\kappa_{3 e_{1}}\left(X_{N}\right)\right|}=\sup _{N} a_{N}^{\alpha} N^{-\frac{\alpha(2 m+d)}{2 d}} \text { for } \alpha=1,2,3, \\
& \text { (iii) } \quad \sup _{N} \frac{a_{N}^{\alpha}\left|\kappa_{(4-\alpha) e_{1}+\alpha e_{2}}\left(X_{N}\right)\right|^{3 / 4}}{\left|\kappa_{3 e_{1}}\left(X_{N}\right)\right|}=\sup _{N} a_{N}^{\alpha} N^{-\frac{3(\alpha+1)}{4}\left(\frac{2 m+d}{2 d}\right)} \\
& \text { for } \alpha=0,1,2,3,4 .
\end{aligned}
$$

If we take $a_{N}=N^{k\left(\frac{2 m+d}{2 d}\right)}$ for $k<\frac{15}{16}$, the right-hand sides in (75) and (77) meet the conditions (ii) and (iii) in Theorem 5. Moreover, the inequality

$$
\bar{\Phi}(z) \leq \frac{1}{z \sqrt{2 \pi}} e^{-\frac{z^{2}}{2}}
$$

proves that the condition (37)

$$
\sup _{N} \frac{\bar{\Phi}\left(a_{N}\right)}{M_{N}}<\infty
$$

is satisfied. Thus the proof of this theorem is completed.

Remark 1. In Theorem 7 of the paper [12], the authors obtain an optimal bound (73) under the assumption $m_{1} \geq \frac{3 m}{2}-\frac{d}{4}$. However, in this paper, we assume that $m_{1} \geq \frac{4 m}{3}-\frac{d}{3}$. Note that

$$
\frac{3 m}{2}-\frac{d}{4}>\frac{4 m}{3}-\frac{d}{3}
$$

This fact shows that the result given in this paper is an extension of the previous result. 


\subsection{Ornstein-Uhlenbeck Process}

In this section, we find an optimal rate of convergence of the distribution of the MLE $\hat{\theta}_{T}$ of the unknown parameter $\theta \in \Theta \subseteq \mathbb{R}^{+}$based on the observation $X=\left\{X_{t}, 0 \leq t \leq T\right\}$ given by

$$
d X_{t}=-\theta X_{t} d t+d W_{t}, X_{0}=0,0 \leq t \leq T,
$$

where $\left\{W_{t}, t \geq 0\right\}$ is a standard Brownian motion. Define kernels $f_{2}(s, t)$ and $g_{2}(s, t)$ for $t, s \in[0, T]$ as

$$
\begin{aligned}
f_{2}(s, t) & =e^{-\theta|t-s|}, \\
g_{2}(u, v) & =\frac{e^{\theta(u+v)}}{2 \theta}\left(e^{-2 \theta(u \vee v)}-e^{-2 \theta T}\right) .
\end{aligned}
$$

When the process $\left\{X_{t}, 0 \leq t \leq T\right\}$ can be observed, we can write

$$
\sqrt{\frac{T}{2 \theta}}\left(\hat{\theta}_{T}-\theta\right)=\frac{-F_{T}}{\bar{G}_{T}+Q_{T}},
$$

where $F_{T}$ and $\bar{G}_{T}$ are the double stochastic integrals

$$
F_{T}=\sqrt{\frac{\theta}{2 T}} I_{2}\left(f_{2}\right), \quad \bar{G}_{T}=\frac{2 \theta}{T} I_{2}\left(g_{2}\right)
$$

and

$$
Q_{T}=1-\frac{1}{2 \theta T}\left(1-e^{-2 \theta T}\right)
$$

Let us set $Y_{T}=\left(F_{T}, \bar{G}_{T}\right)$.

Lemma 7. For $\beta=2,3,4$, we have that for $\alpha=0,1, \ldots, \beta$,

$$
\kappa_{(\beta-\alpha) e_{1}+\alpha e_{2}}\left(Y_{T}\right)=c_{\theta, \alpha, \beta} T^{-\frac{\beta+\alpha-2}{2}}+o\left(T^{-\frac{\beta+\alpha-2}{2}}\right) .
$$

Proof. For the proof, we will use the method in the proof of Lemma 6. Since $e^{-\theta|t-s|}=$ $e^{\theta(t+s)-2 \theta(s \vee t)}$, the kernel function $g_{2}$ in (79) can be written as

$$
g_{2}(s, t)=\frac{1}{2 \theta}\left(e^{-\theta|t-s|}-e^{-\theta(2 T-t-s)}\right) .
$$

Obviously, $e^{-\theta|t-s|} \geq e^{-\theta(2 T-t-s)}$ for all $s, t \in[0, T]$. This implies the fact that it is sufficient to consider the term $e^{-\theta|t-s|}$ in $g_{2}(s, t)$ as the main term of the asymptotics of $\kappa_{(\beta-\alpha) e_{1}+\alpha e_{2}}\left(Y_{T}\right)$ for $\alpha=0,1, \ldots, \beta$, where $\beta=2,3,4$. Hence the main term is given by

$$
\begin{aligned}
Q_{N}(\alpha, \beta) & =\left(\frac{\theta}{2}\right)^{\frac{\beta+\alpha}{2}} T^{-\frac{\alpha+\beta}{2}} \int_{[0, T]^{\beta}} \prod_{i=1}^{\beta} e^{-\theta\left|z_{i}-z_{i+1}\right|} d z_{1} \ldots d z_{\beta} \\
& =\beta !\left(\frac{\theta}{2}\right)^{\frac{\beta+\alpha}{2}} T^{-\frac{\alpha+\beta}{2}} \int_{\left\{0 \leq z_{1} \leq \ldots \leq z_{\beta} \leq T\right\}} e^{2 \theta\left(z_{\beta}-z_{1}\right)} d z_{1} \ldots d z_{4} \\
& =c T^{-\frac{\alpha+\beta-2}{2}}+o\left(T^{-\frac{\alpha+\beta-2}{2}}\right) .
\end{aligned}
$$

Here we define $z_{\beta+1}=z_{1}$. Therefore, the proof of this lemma is completed.

By using Theorem 5, we obtain an optimal Berry-Esseen bound for the sequence $\left\{\sqrt{\frac{T}{2 \theta}}\left(\hat{\theta}_{T}-\theta\right), T \geq 0\right\}$. 
Theorem 8 (Optimal bound). For sufficiently large $T>0$, there exist constants $0<c_{\theta}<C_{\theta}<$ $\infty$ such that

$$
c_{\theta} \frac{1}{\sqrt{T}} \leq \sup _{z \in \mathbb{R}}\left|\mathbb{P}\left(\sqrt{\frac{T}{2 \theta}}\left(\hat{\theta}_{T}-\theta\right) \leq z\right)-\mathbb{P}(Z \leq z)\right| \leq C_{\theta} \frac{1}{\sqrt{T}}
$$

Proof. For sufficiently large $n_{0}>0$, we have, from Lemma 7 , that

$$
\begin{gathered}
\text { (i) } \sup _{T \geq n_{0}} \frac{\left|\kappa_{2 e_{2}}\left(Y_{T}\right)\right|}{\left|\kappa_{e_{1}+e_{2}}\left(Y_{T}\right)\right|}=c \sup _{T \geq n_{0}} \frac{1}{\sqrt{T}}<\infty, \\
\text { (ii) } \sup _{T \geq n_{0}} \frac{a_{T}^{\alpha}\left|\kappa_{(3-\alpha) e_{1}+\alpha e_{2}}\left(Y_{T}\right)\right|}{\left|\kappa_{3 e_{1}}\left(Y_{T}\right)\right|}=\sup _{T \geq n_{0}} a_{T}^{\alpha} T^{-\frac{\alpha}{2}} \text { for } \alpha=1,2,3, \\
\text { (iii) } \quad \sup _{T \geq n_{0}} \frac{a_{T}^{\alpha}\left|\kappa_{(4-\alpha) e_{1}+\alpha e_{2}}\left(Y_{T}\right)\right|^{3 / 4}}{\left|\kappa_{3 e_{1}}\left(Y_{T}\right)\right|}=\sup _{T \geq n_{0}} a_{T}^{\alpha} T^{-\frac{2+3 \alpha}{8}} \\
\text { for } \alpha=0,1,2,3,4 .
\end{gathered}
$$

If we take $a_{T}=T^{k / 2}$ for $k<\frac{7}{8}$, the right-hand sides in (87) and (89) satisfy the conditions (ii) and (iii) in Theorem 5, and obviously,

$$
\sup _{T \geq n_{0}} \frac{\bar{\Phi}\left(a_{T}\right)}{M_{T}}<\infty .
$$

Hence the proof of this theorem is completed.

\section{3. $\alpha$-Brownian Bridge}

In [19], the authors find an optimal rate of convergence of the distribution of the MLE $\hat{\alpha}_{t}$ of the unknown parameter $\alpha \in(1 / 2, \infty)$ based on the observation $X=\left\{X_{t}, 0 \leq t \leq T\right\}$ given by the $\alpha$-Brownian bridge

$$
d X_{t}=-\alpha \frac{X_{t}}{T-t} d t+d W_{t}, X_{0}=0,0 \leq t<T,
$$

where $\left\{W_{t}, t \geq 0\right\}$ is a standard Brownian motion. Here we will derive an optimal Berry-Esseen bound using our result, Theorem 5. Define kernels $f_{t}(u, v)$ and $g_{t}(u, v)$ for $u, v \in[0, t]$ as

$$
\begin{aligned}
f_{t}(u, v)= & \frac{\sqrt{2 \alpha-1}}{\sqrt{|\log (T-t)|}}(T-(u \vee v))^{\alpha-1}(T-(u \wedge v))^{-\alpha} \mathbf{1}_{[0, t]^{2}}(u, v), \\
g_{t}(u, v)= & \frac{(T-u)^{-\alpha}(T-v)^{-\alpha}}{|\log (T-t)|}\left[(T-(u \vee v))^{2 \alpha-1}\right. \\
& \left.-(T-t)^{2 \alpha-1}\right] \mathbf{1}_{[0, t]^{2}}(u, v) .
\end{aligned}
$$

Using the above kernel functions (90) and (91), we can write

$$
\sqrt{\frac{|\log (T-t)|}{2 \alpha-1}}\left(\alpha-\hat{\alpha}_{t}\right)=\frac{I_{2}\left(f_{t}\right)}{I_{2}\left(g_{t}\right)+b_{t}},
$$

where $b_{t}$ is given by

$$
\begin{aligned}
b_{t}=1+ & \frac{\log (T)}{|\log (T-t)|}-\frac{1}{(2 \alpha-1)|\log (T-t)|} \\
& \times\left(1-\left(\frac{T-t}{T}\right)^{2 \alpha-1}\right) .
\end{aligned}
$$


Let us set $D_{t}=\left(I_{2}\left(f_{t}\right), I_{2}\left(g_{t}\right)\right)$ for $t \in[0, T)$.

Lemma 8. For $p=2,3,4$, we have that for $q=0,1, \ldots, p$,

$$
\kappa_{(p-q) e_{1}+q e_{2}}\left(D_{t}\right)=\frac{c_{\alpha, p, q}}{|\log (T-t)|^{\frac{p+q-2}{2}}}+o\left(\frac{1}{|\log (T-t)|^{\frac{p+q-2}{2}}}\right) .
$$

Proof. The proof of this lemma can be done by a straightforward adaptation of the computations for the proof of Lemma 6. First, note that

$$
(T-(u \vee v))^{\alpha-1}(T-(u \wedge v))^{-\alpha}=(T-u)^{-\alpha}(T-v)^{-\alpha}(T-(u \vee v))^{2 \alpha-1} .
$$

From this, the kernel function $g_{t}$ in (91) can be written as

$$
\begin{aligned}
g_{t}(u, v)= & \frac{2}{\sqrt{(2 \alpha-1)|\log (T-t)|}} f_{t}(u, v) \\
& -\frac{1}{|\log (T-t)|} h_{t}(u, v),
\end{aligned}
$$

where $h_{t}(u, v)$ is given by

$$
\left.h_{t}(u, v)=(T-u)^{-\alpha}(T-v)^{-\alpha}(T-t)^{2 \alpha-1}\right] \mathbf{1}_{[0, t]^{2}}(u, v) .
$$

Since $(T-(u \vee v))^{2 \alpha-1} \geq(T-t)^{2 \alpha-1}$ for all $u, v \in[0, t]$, it is sufficient to consider only the first term of $g_{2}(u, v)$ in (95) as the main term of the asymptotics of $\kappa_{(p-q) e_{1}+q e_{2}}\left(D_{t}\right)$ for $q=0,1, \ldots, p$, where $p=2,3,4$. Hence a direct computation yields that the asymptotics of the main term is given by

$$
\begin{aligned}
Q_{t}(p, q)= & (2 \alpha-1)^{\frac{p-q}{2}} \frac{1}{|\log (T-t)|^{\frac{p+q}{2}}} \int_{[0, T]^{p}} \prod_{i=1}^{p}\left(T-\left(u_{i} \vee u_{i+1}\right)\right)^{q-1} \\
& \times\left(T-\left(u_{i} \wedge u_{i=1}\right)\right)^{-\alpha} d z_{1} \ldots d z_{\beta} \\
= & \frac{c_{\alpha, p, q}}{|\log (T-t)|^{\frac{p+q-2}{2}}}+o\left(\frac{1}{|\log (T-t)|^{\frac{p+q-2}{2}}}\right) .
\end{aligned}
$$

Here we define $z_{\beta+1}=z_{1}$. Therefore, the proof of this lemma is completed.

By using Theorem 5, we obtain an optimal Berry-Esseen bound for the distribution of (92).

Theorem 9 (Optimal bound). For all $t \in[0, T)$ sufficiently near $T>0$ and $\alpha>1 / 2$, there exist constants $0<c_{\alpha}<C_{\alpha}<\infty$ such that

$$
\begin{gathered}
\frac{c_{\alpha}}{\sqrt{|\log (T-t)|}} \leq \sup _{z \in \mathbb{R}}\left|\mathbb{P}\left(\sqrt{\frac{|\log (T-t)|}{2 \alpha-1}}\left(\alpha-\hat{\alpha}_{t}\right) \leq z\right)-\mathbb{P}(Z \leq z)\right| \\
\leq \frac{C_{\alpha}}{\sqrt{|\log (T-t)|}}
\end{gathered}
$$


Proof. Let $T_{0}$ be sufficiently near $T>0$. Then we have, from Lemma 8 , that

$$
\begin{gathered}
\text { (i) } \sup _{t \geq T_{0}} \frac{\left|\kappa_{2 e_{2}}\left(D_{t}\right)\right|}{\left|\kappa_{e_{1}+e_{2}}\left(D_{t}\right)\right|}=c \sup _{t \geq T_{0}} \frac{1}{\sqrt{|\log (T-t)|}}<\infty, \\
\text { (ii) } \sup _{t \geq T_{0}} \frac{a_{t}^{q}\left|\kappa_{(3-q) e_{1}+\alpha e_{2}}\left(D_{t}\right)\right|}{\left|\kappa_{3 e_{1}}\left(D_{t}\right)\right|}=\sup _{t \geq T_{0}} \frac{a_{t}^{q}}{|\log (T-t)|^{\frac{q}{2}}} \text { for } q=1,2,3, \\
\text { (iii) } \quad \sup _{t \geq T_{0}} \frac{a_{t}^{q}\left|\mathcal{K}_{(4-q) e_{1}+\alpha e_{2}}\left(D_{t}\right)\right|^{3 / 4}}{\left|\kappa_{3 e_{1}}\left(D_{t}\right)\right|}=\sup _{t \geq T_{0}} \frac{a_{t}^{q}}{|\log (T-t)|^{\frac{2+3 q}{8}}} \\
\text { for } q=0,1,2,3,4 .
\end{gathered}
$$

If we take $a_{t}=|\log (T-t)|^{k / 2}$ for $k<\frac{7}{8}$, the right-hand sides in (99) and (101) satisfy the conditions (ii) and (iii) in Theorem 5. A direct computation shows that

$$
M_{t}=\frac{1}{\sqrt{|\log (T-t)|}}+o\left(\frac{1}{\sqrt{|\log (T-t)|}}\right),
$$

so that using the inequality (77) gives

$$
\begin{aligned}
\sup _{t \geq T_{0}} \frac{\bar{\Phi}\left(a_{t}\right)}{M_{t}} \leq & \frac{1}{\sqrt{2 \pi}} \sup _{t \geq T_{0}} \frac{e^{-\frac{a_{t}^{2}}{2}}}{a_{t} M_{t}} \\
= & \frac{1}{\sqrt{2 \pi}} \sup _{t \geq T_{0}}|\log (T-t)|^{\frac{1-k}{2}} \exp \left(-\frac{|\log (T-t)|^{k}}{2}\right) \\
& <\infty .
\end{aligned}
$$

The proof of this theorem is completed.

\section{Conclusions and Future Works}

This paper developed a method to obtain an optimal Berry-Esseen bound for $F / G$, the form in which the estimator of a parameter in SDEs or SPDEs take place. To this end, we used the Edgeworth expansion of $F / G$ derived in this paper. In order to achieve the optimal bound, all we have to do is to show that the conditions found in this paper are satisfied. The significant feature of our method is that the conditions of proof are met in many estimation problems of a parameter in SDEs or SPDEs. Moreover, we emphasize that Theorem 5 is an extension of the optimal bound given in (4) mentioned in the introduction, so it has drawn very meaningful results by itself. Future works will be carried out in two directions: (1) Applying our method to previous works such as [22,23], etc.; (2) finding new problems to apply our method to.

Author Contributions: Conceptualization, Y.-T.K. and H.-S.P.; methodology, Y.-T.K.; writing and original draft preparation, Y.-T.K. and H.-S.P.; co-review and validation, H.-S.P. Both authors have read and agreed to the published version of the manuscript.

Funding: This research was supported by Hallym University Research Fund (HRF-202108-003).

Institutional Review Board Statement: Not applicable.

Informed Consent Statement: Not applicable.

Data Availability Statement: Not applicable.

Acknowledgments: We are grateful for the helpful comments of referees which helped us improve the paper.

Conflicts of Interest: The authors declare no conflict of interest. 


\section{References}

1. Nualart, D.; Peccati, G. Central limit theorems for sequences of multiple stochastic integrals. Ann. Prob. $2015,33,177-193$. [CrossRef]

2. Nourdin, I.; Peccati, G. Stein's method on Wiener Chaos. Probab. Theory Relat. Fields 2009, 145, 75-118. [CrossRef]

3. Nourdin, I. Lectures on Gaussian approximations with Malliavin calculus. In Proceedings of the Séminaire de Probabilités XLV; Lecture Notes in Mathematics; Springer: Berlin/Heidelberg, Germany, 2013; Volume 2078. [CrossRef]

4. Nourdin, I.; Peccati, G. Stein's method meets Malliavin calculus: A short survey with new estimates. In Recent Development in Stochastic Dynamics and Stochasdtic Analysis; World Scientific Publishing: Hackensack, NJ, USA, 2010; pp. 207-236.

5. Nourdin, I. Peccati, G. Normal approximations with Malliavin calculus: From Stein's method to universality. In Cambridge Tracts in Mathematica; Cambridge University Press: Cambridge, UK, 2012; Volume 192.

6. Nualart, D. Malliavin calculus and related topics. In Probability and Its Applications, 2nd ed.; Springer: Berlin, Germany, 2006.

7. Chen, L.H.Y.; Goldstein, L.; Shao, Q.-M. Normal Apprtoximation by Stein's Method; Probability and Its Applications; Springer: Heidelberg, Germany; New York, NY, USA, 2011.

8. Stein, C. A bound for the error in the normal approximation to the distribution of a sum of dependent random variables. In Proceedings of the Sixth Berkeley Symposium on Mathematical Statistics and Probability, Volume 2: Probability Theory; University of California Press: Berkeley, CA, USA, 1972; pp. 583-602.

9. Stein, C. Approximate Computation of Expectations; IMS: Hayward, CA, USA, 1986.

10. Biermé, H.; Bonami, A.; Nourdin, I.; Peccati, G. Optimal Berry-Esseen rates on the Wiener space: The barrier of third and fourth cumulants. ALEA Lat. Am. J. Probab. Math. Stat. 2012, 9, 473-500.

11. Nourdin, I. Peccati, G. The optimal fourth moment theorem. Proc. Am. Math. Soc. 2015, 143, 3123-3133. [CrossRef]

12. Kim, Y.T.; Park, H.S. Optimal Berry-Esseen bound for statistical estimations and its application to SPDE. J. Multi. Anal. 2017, 155, 284-304. [CrossRef]

13. Kim, Y.T.; Park, H.S. An Edeworth expansion for functionals of Gaussian fields and its applications. Stoch. Process. Their Appl. 2018, 44, 312-320.

14. Hall, P. The Bootstrap and Edgeworth Expansion; Springer Series in Statistics; Springer: New York, NY, USA, 1992.

15. McCullagh, P. Tensor methods in statistics. In Monographs on Statistics and Applied Probability; Chapman \& Hall: London, UK, 1987.

16. Nourdin, I. Peccati, G. Cumulants on the Wiener space. J. Funct. Anal. 2010, 258, 3775-3791. [CrossRef]

17. Leonov, V.P.; Shiryaev, A.N. On the method of calculations of semi-invariants. Theory Probab. Its Appl. 1959, 4, 319-329. [CrossRef]

18. Peccati, G.; Taqqu, M. Moments, cumulants and diagram formulae for non-linear functionals of random measures. arXiv 2008, arXiv:0811.1726.

19. Es-Sebaiy, K.; Moustaaid, J. Optimal Berry-Esséen bound for Maximum likelihood estimation of the drift parameter in $\alpha$-Brownian bridge. J. Korean Stat. Soc. 2021, 50, 403-418. [CrossRef]

20. Kim, Y.T.; Park, H.S. Optimal Berry-Esseen bound for an estimator of parameter in the Ornstein-Uhlenbeck process. J. Korean Statist. Soc. 2017, 46, 413-425. [CrossRef]

21. Huebner, M.; Rozovskii, B.L. On asymptotic properties of maximum likelihood estimators for parabolic stochastic PDE's. Probab. Theory Relat. Fields 1995, 103, 143-163. [CrossRef]

22. Chen, Y.; Kuang, N.; Li, Y. Berry-Esséen bound for the parameter estimation of fractional Ornstein-Uhlenbeck processes. Stochastics Dyn. 2020, 20, 2050023. [CrossRef]

23. Es-Sebaiy, K. Berry-Esséen bounds for the least squares estimator for discretely observed fractional Ornstein-Uhlenbeck processes. Stat. Prob. Lett. 2012, 83, 2372-2385. [CrossRef] 